TRANSACTIONS OF THE

AMERICAN MATHEMATICAL SOCIETY

Volume 357, Number 2, Pages 709-734

S 0002-9947(04)03482-8

Article electronically published on July 16, 2004

\title{
THE RELATIONSHIP BETWEEN HOMOLOGICAL PROPERTIES AND REPRESENTATION THEORETIC REALIZATION OF ARTIN ALGEBRAS
}

\author{
OSAMU IYAMA
}

\begin{abstract}
We will study the relationship of quite different objects in the theory of artin algebras, namely Auslander-regular rings of global dimension two, torsion theories, $\tau$-categories and almost abelian categories. We will apply our results to characterization problems of Auslander-Reiten quivers.
\end{abstract}

0.1. There exists a bijection between equivalence classes of Krull-Schmidt categories $\mathcal{C}$ with additive generators $M$ and Morita-equivalence classes of semiperfect rings $\Gamma$, which is given by $\mathcal{C} \mapsto \mathcal{C}(M, M)$ and the converse is given by $\Gamma \mapsto \operatorname{pr} \Gamma$ for the category $\operatorname{pr} \Gamma$ of finitely generated projective $\Gamma$-modules. Although this bijection itself is rather formal, it will be very fruitful to study the relationship between (A)-(D) below. The object of this paper is to study it under the assumption that $\Gamma$ is an artin algebra.

(A) Homological properties for $\Gamma$.

(B) Representation theoretic realization of $\mathcal{C}$.

(C) Categorical properties for $\mathcal{C}$.

(D) Combinatorial properties for the $\mathrm{AR}$ quiver $\mathbb{A}(\mathcal{C})$.

For $(\mathrm{A})$, we will study a property of the selfinjective resolution of $\Gamma$ which is called the $(l, n)$-conditions ( $\$ 1.1)$ and generalizes both the Auslander conditions [Bj] and the dominant dimension [T]. For (B), we will study the existence of an equivalence between $\mathcal{C}$ and a torsionfree class of $\bmod \Lambda$ over an artin algebra $\Lambda(\S 1.2, \S 2.2)$, where such a subcategory is very popular in the representation theory of artin algebras $[\mathrm{Ha}$, $\overline{\mathrm{As}}$. For $(\mathrm{C})$, we will treat a class of additive categories which are called $\tau$-categories $(\$ 1.3)$ and introduced in [I3]. $\tau$-categories are additive categories with generalized almost split sequences, and our motivation and definition were rather different from the work of Auslander and Smalø in [AS] since we aimed to treat categories which can be far from abelian, for example, mesh categories of translation quivers $(\S 1.3 .2(3))$. Nevertheless our result Theorem 2.1 asserts that some $\tau$-categories are realized as torsionfree classes over artin algebras, and they form almost abelian categories ( $(1.5)$. For (D), we will study a combinatorial invariant $\mathbb{A}(\mathcal{C})$ of a $\tau$-category $\mathcal{C}$ called the $A R$ (= Auslander-Reiten) quiver $(\S 4.1)$. Some results in this paper were already announced in [I6, 7.4] without proof.

Received by the editors July 9, 2002 and, in revised form, July 31, 2003.

2000 Mathematics Subject Classification. Primary 16E65; Secondary 16G70. 
0.2. Background. In $[\mathrm{A}$, Auslander obtained a quite remarkable theorem which asserts that there exists a bijection between Morita-equivalence classes of representation-finite artin algebras $\Lambda$ and those of Auslander algebras $\Gamma$, which is an artin algebra with gl.dim $\Gamma \leq 2$ and $\operatorname{dom} \cdot \operatorname{dim} \Gamma \geq 2$. Then $\bmod \Lambda$ is equivalent to $\operatorname{pr} \Gamma$, and this correspondence gives a prototype of our study. It relates a representation theoretic realization (B) of the category $\mathcal{C}=\operatorname{pr} \Gamma$ to a homological property (A) of $\Gamma$, and it will be suggestive that Auslander algebras form a special class of Auslander-regular rings $\Gamma$ with gl.dim $\Gamma \leq 2$. In [FGR, [Bj], [AR2], [C], and so on, Auslander-regular rings, more generally Auslander-Gorenstein rings, are studied as a non-commutative analogy of commutative Gorenstein rings motivated by the classical results of Bass $\mathrm{B}$.

Later, Auslander and Reiten AR1] obtained the existence theorem of almost split sequences, which is one of the most important theorems in the representation theory of algebras ARS. This theorem gives a categorical property $(\mathrm{C})$ of $\bmod \Lambda$ over an artin algebra $\Lambda$, which means that "mod $\Lambda$ forms a $\tau$-category" in our context. Although this theorem is a great achievement of general theory of homological algebra, it can be proved easily for the special case when $\Lambda$ is representation-finite, and this observation seems to lead them to the general existence theorem. Moreover, the theorem for the representation-finite case has its own importance. In terms of the corresponding Auslander algebra $\Gamma$, it means that the functor $\operatorname{Ext}_{\Gamma}^{2}(, \Gamma)$ : $\bmod \Gamma \leftrightarrow \bmod \Gamma^{o p}$ gives a duality between simple $\Gamma$-modules $L$ with $\operatorname{pd} L=2$ and that of $\Gamma^{o p}$. We can naturally generalize this duality to arbitrary AuslanderGorenstein rings [9] (see $§ 3.6 .3$ below).

By applying Auslander's correspondence theorem and extending some aspects in [BG], Igusa-Todorov and Brenner gave (distinct) characterizations of AR quivers of representation-finite artin algebras $[\mathrm{IT} 3, \mathrm{Br}$. These are nothing but the combinatorial properties $(\mathrm{D})$ of $\mathbb{A}(\mathcal{C})$. Recently, inspired by the work of Igusa-Todorov, the author introduced $\tau$-categories and successfully applied them to characterize AR quivers of representation-finite orders [I3], I4], [I5]. We shall see that $\tau$-categories give a powerful tool for our problem.

0.3. Our results. Our first theorem (Theorem 2.1) gives the relationship between (A)-(D) in $\S 0.1$ for more general classes of algebras than those in $\S 0.2$, namely the conditions below are equivalent for an artin algebra $\Gamma$ and $\mathcal{C}=\operatorname{pr} \Gamma$.

(A) $\Gamma$ satisfies gl.dim $\Gamma \leq 2$ and the two-sided $(2,2)$-condition $(\S 1.1)$.

(B) $\mathcal{C}$ is equivalent to a faithful torsionfree class over an artin algebra $(\S 1.2)$.

(C) $\mathcal{C}$ is a strict $\tau$-category $(\S 1.3)$.

(D) $\mathcal{C}$ is a $\tau$-category with a right additive function on $\mathbb{A}(\mathcal{C})(\S 1.3 .1)$.

Next we will study Auslander-regular rings $\Gamma$ with gl.dim $\Gamma \leq 2$, which forms a special class of algebras in (A) above. Our second theorem (Theorem 3.1) gives the corresponding objects in (B)-(D) to such $\Gamma$, namely hereditary torsionfree classes $(\S 1.2)$ correspond for $(\mathrm{B})$, strict $\tau$-categories with "Nakayama pairs" (§1.4) correspond for $(\mathrm{C})$, and $\tau$-categories with additive functions on $\mathbb{A}(\mathcal{C})(\S 1.3 .1)$ correspond for (D). The concept of Nakayama pairs was introduced in [I4 to characterize AR quivers of representation-finite orders, and they were essentially used also in IgusaTodorov's theorem ( $\$ 0.2)$. The concept of additive functions often appeared in representation theory (see $\S 4$ ), for example, Brenner's theorem ( $\S 0.2)$ and Rump's recent characterization of AR quivers of representation-finite orders [R5]. Moreover, we will study two special classes of Auslander-regular rings $\Gamma$ with gl.dim $\Gamma \leq 2$, and 
give the corresponding objects in (B)-(D) again. One is Auslander algebras (§3.3) which gave a prototype of our study, and we will prove very clearly Auslander's correspondence theorem, Igusa-Todorov's theorem and Brenner's theorem explained in $\S 0.2$. Another is diagonal Auslander-regular rings $\Gamma$ with gl.dim $\Gamma \leq 2(\S 3.4)$, which are closely related to the category $\bmod { }_{s p} \Lambda$ of modules with projective socles $[$, and we will generalize a result of Ringel-Vossieck [RV]. The connection of several known results will be understood very clearly in our functorial and homological viewpoint of this paper. As we shall see in examples in $\S 4.5$, our characterizations of AR quivers in Theorem 4.4 are very simple and can be checked easily.

We will discuss properties of $\Gamma$ with gl.dim $\Gamma \leq 2$ and the two-sided $(2,2)$ condition, namely symmetry in $\S 3.6 .1$, duality in $\S 3.6 .3$ and the quasi-Koszul property [GM] of Green-Martinez in $\S 2.5$. In the final section, $\S 5$, we will study the rejection theory of faithful torsionfree classes over artin algebras. The rejection theory of $\tau$-categories was studied in [14, 4] as a wide generalization of the DK (=Drozd-Kirichenko) Rejection Lemma which was fundamental in the theory of Bass orders ([DKR], [Ro], $[\mathrm{HN}])$. They played a crucial role in characterizing AR quivers of representation-finite orders [15], and recently they were applied to prove Solomon's second conjecture on zeta functions of orders [17] and finiteness of representation dimension of artin algebras [18].

All artin algebras $\Gamma$ in (A) studied in this paper satisfy gl.dim $\Gamma \leq 2$. It will be very interesting to generalize our results to artin algebras $\Gamma$ with gl.dim $\Gamma \geq 3$.

Finally, notice that W. Rump's recent work [R1]-R5] on almost abelian categories $(\S 1.5)$ and $\tau$-categories has a strong relationship with our study.

\section{Preliminaries}

In this paper, any module is assumed to be a left module. For a ring $\Lambda$, we denote by $\bmod \Lambda$ the category of finitely generated $\Lambda$-modules, by $\operatorname{pr} \Lambda(\operatorname{resp} . \operatorname{sim} \Lambda)$ the category of finitely generated projective (resp. simple) $\Lambda$-modules, by $J_{\Lambda}$ the Jacobson radical of $\Lambda$, by $\widehat{()}$ the functor $\operatorname{Hom}_{\Lambda}(, \Lambda): \bmod \Lambda \leftrightarrow \bmod \Lambda^{o p}$, by $0 \rightarrow X \rightarrow I_{\Lambda}^{0}(X) \rightarrow I_{\Lambda}^{1}(X) \rightarrow \cdots$ a minimal injective resolution of a $\Lambda$-module $X$, and by $\operatorname{pd} X$ (resp. fd $X$, id $X$ ) the projective (resp. flat, injective) dimension of a $\Lambda$-module $X$. We denote by $\underline{\bmod } \Lambda$ the stable category, by $\Omega: \underline{\bmod } \Lambda \rightarrow \underline{\bmod } \Lambda$ the syzygy functor, and by $\operatorname{Tr}: \underline{\bmod } \Lambda \rightarrow \underline{\bmod } \Lambda^{o p}$ the transpose functor $[\overline{\mathrm{AB}}$. When $\Lambda$ is an artin algebra over $R$, we denote by ( $)^{*}$ the duality $\operatorname{Hom}_{R}\left(, I_{R}^{0}\left(R / J_{R}\right)\right)$ : $\bmod \Lambda \leftrightarrow \bmod \Lambda^{o p}$, and by in $\Lambda$ the category of finitely generated injective $\Lambda$ modules. For an additive category $\mathcal{C}$ and $X \in \mathcal{C}$, we denote by add $X$ the full subcategory of $\mathcal{C}$ consisting of direct summands of $X^{n}(n>0)$. We call $X$ an additive generator of $\mathcal{C}$ if $\mathcal{C}=\operatorname{add} X$ holds.

1.1. Let $\Gamma$ be a noetherian ring. We denote by grade $L:=\inf \left\{i \geq 0 \mid \operatorname{Ext}_{\Gamma}^{i}(L, \Gamma) \neq\right.$ $0\}$ (resp. s.grade $L:=\inf \{$ grade $M \mid M \subseteq L\}, \operatorname{rg} \cdot \operatorname{grade} L:=\inf \left\{i>0 \mid \operatorname{Ext}_{\Gamma}^{i}(L, \Gamma) \neq\right.$ $0\}$ ) the grade (resp. strong grade, reduced grade) of $L \in \bmod \Gamma$. For any $n \geq 0$, the full subcategory $\{L \mid$ s.grade $L \geq n\}$ of $\bmod \Gamma$ is abelian and closed under subfactor modules and extensions. For $l, n>0$, we say that $\Gamma$ satisfies the $(l, n)$-condition if the following equivalent conditions are satisfied [I5, 6.1].

(i) fd $I_{\Gamma}^{i}(\Gamma)<l$ holds for any $i(0 \leq i<n)$.

(ii) s.grade $\operatorname{Ext}_{\Gamma}^{l}(L, \Gamma) \geq n$ holds for any $L \in \bmod \Gamma^{o p}$. 
This equivalence simplifies the equivalence of (a) and (c) in the famous theorem of [FGR, 3.7] and that of (b) and (d) in [AR3, 0.1]. For an artin algebra $\Gamma$, the $(l, n)$-condition is equivalent to the condition that s.grade $\operatorname{Ext}_{\Gamma}^{l}(L, \Gamma) \geq n$ holds for any simple $\Gamma^{o p}$-module $L$ [AR2]. We say that $\Gamma$ satisfies the $(l, n)^{o p}$-condition if $\Gamma^{o p}$ satisfies the $(l, n)$-condition.

Put dom.dim $\Gamma:=\inf \left\{i \geq 0 \mid \mathrm{fd} I_{\Gamma}^{i}(\Gamma) \neq 0\right\}[\mathrm{T}$, which is the maximal number $n$ such that $\Gamma$ satisfies the $(1, n)$-condition. We call $\Gamma n$-Gorenstein if $\mathrm{fd} I_{\Gamma}^{i}(\Gamma) \leq i$ holds for any $i(0 \leq i<n)$ [FGR], or equivalently, $\Gamma$ satisfies the $(l, l)$-condition for any $l(0<l \leq n)$. We call $\Gamma$ Auslander-regular (resp. Auslander-Gorenstein) if gl.dim $\Gamma<\infty\left(\right.$ resp. $\operatorname{id}_{\Gamma} \Gamma<\infty$ and id $\left.\Gamma_{\Gamma}<\infty\right)$ and $\Gamma$ is $n$-Gorenstein for any $n$ [C]. It is well known that $\operatorname{dom} \cdot \operatorname{dim} \Gamma=\operatorname{dom} \cdot \operatorname{dim} \Gamma^{o p}$ holds [H2], and $\Gamma$ is $n$-Gorenstein if and only if $\Gamma^{o p}$ is also [FGR, 3.7]. These left-right symmetries were generalized to the $(l, n)$-condition in [I9] (see 3.6.1 below), although the $(l, n)$-condition itself is not left-right symmetric (2.1.1(2)).

1.2. Let $\Lambda$ be an artin algebra. We call a full subcategory $\mathcal{C}$ of $\bmod \Lambda$ a torsionfree (resp. torsion) class if it is closed under submodules (resp. factor modules) and extensions $\mathrm{As}$. For a collection $\mathbf{S}$ of $\Lambda$-modules, define full subcategories of $\bmod \Lambda$ by $\mathbf{S}^{\perp}:=\left\{X \mid \operatorname{Hom}_{\Lambda}(Y, X)=0\right.$ for any $\left.Y \in \mathbf{S}\right\}$ and ${ }^{\perp} \mathbf{S}:=\left\{X \mid \operatorname{Hom}_{\Lambda}(X, Y)=0\right.$ for any $Y \in \mathbf{S}\}$. We call a pair $(\mathcal{T}, \mathcal{F})$ of full subcategories of $\bmod \Lambda$ a torsion theory on $\bmod \Lambda$ if it satisfies the following equivalent conditions:

(i) $\mathcal{F}=\mathcal{T}^{\perp}$ and $\mathcal{T}={ }^{\perp} \mathcal{F}$.

(ii) $\mathcal{F}$ is a torsionfree class and $\mathcal{T}=\perp \mathcal{F}$.

(iii) $\mathcal{T}$ is a torsion class and $\mathcal{F}=\mathcal{T}^{\perp}$.

(iv) The inclusion $\mathcal{F} \rightarrow \bmod \Lambda$ has a left adjoint $\mathbb{F}: \bmod \Lambda \rightarrow \mathcal{F}$ with a unit $\alpha$ and the inclusion $\mathcal{T} \rightarrow \bmod \Lambda$ has a right adjoint $\mathbb{T}: \bmod \Lambda \rightarrow \mathcal{T}$ with a counit $\beta$ such that $0 \rightarrow \mathbb{T} \stackrel{\beta}{\rightarrow} 1_{\bmod \Lambda} \stackrel{\alpha}{\rightarrow} \mathbb{F} \rightarrow 0$ is exact.

We call a torsion theory $(\mathcal{T}, \mathcal{F})$ (resp. torsion class $\mathcal{T}$, torsionfree class $\mathcal{F}$ ) faithful if $\Lambda \in \mathcal{F}$, cofaithful if $\Lambda^{*} \in \mathcal{T}$, hereditary if $\mathcal{T}$ is closed under submodules, and cohereditary if $\mathcal{F}$ is closed under factor modules. If $(\mathcal{T}, \mathcal{F})$ is a hereditary torsion theory, then $\mathcal{F}=\mathbf{S}^{\perp}$ holds for the set $\mathbf{S}$ of simple $\Lambda$-modules in $\mathcal{T}$. The facts below show that the faithfulness is fundamental for torsion theories.

1.2.1. Proposition. Let $(\mathcal{T}, \mathcal{F})$ and $\left(\mathcal{T}_{i}, \mathcal{F}_{i}\right)$ be torsion theories on $\bmod \Lambda$ and $\bmod \Lambda_{i}(i=1,2)$, respectively.

(1) There exists a factor algebra $\Gamma$ of $\Lambda$ such that $\mathcal{F} \subseteq \bmod \Gamma$ and $(\mathcal{T} \cap \bmod \Gamma, \mathcal{F})$ is a faithful torsion theory on $\bmod \Gamma$.

(2) $\mathcal{F}$ is faithful and contravariantly finite if and only if there exists a cotilting $\Lambda$-module $U \in \bmod \Lambda$ with $\operatorname{id} U \leq 1$ such that $\mathcal{T}={ }^{\perp} U$.

(3) Assume that $\left(\mathcal{T}_{i}, \mathcal{F}_{i}\right)$ is faithful for $i=1,2$. Then any equivalence $\mathbb{I}: \mathcal{F}_{1} \rightarrow \mathcal{F}_{2}$ extends uniquely to an equivalence $\bmod \Lambda_{1} \rightarrow \bmod \Lambda_{2}$.

Proof. (1) Put $I:=\bigcap_{X \in \mathcal{F}} \operatorname{ann}_{\Lambda} X$ and $\Gamma:=\Lambda / I$. Then $\mathcal{F} \subseteq \bmod \Gamma$ holds, and there exists a faithful $\Gamma$-module $Y \in \mathcal{F}$. Put $E:=\operatorname{End}_{\Gamma}(Y)$ and take a surjection $f \in \operatorname{Hom}_{E}\left(E^{n}, Y\right)$. Taking $\operatorname{Hom}_{E}(, Y)$, we obtain an injection $(f \cdot) \in$ $\operatorname{Hom}_{\Gamma}\left(\operatorname{End}_{E}(Y), Y^{n}\right)$. Thus $\Gamma \in \mathcal{F}$ holds by $\Gamma \subseteq \operatorname{End}_{E}(Y)$. Obviously $(\mathcal{T} \cap$ $\bmod \Gamma, \mathcal{F})$ forms a torsion theory on $\bmod \Gamma$.

(2) Well known (see [H1], [AS], $\overline{\mathrm{As}}]$ ).

(3) Suppose that $X \in \operatorname{pr} \Lambda_{1}$ satisfies $\mathbb{I} X \notin \operatorname{pr} \Lambda_{2}$. Since $\Lambda_{2} \in \mathcal{F}_{2}$ holds, there exists $f \in \operatorname{Hom}_{\Lambda_{1}}(Y, X)$ such that $\mathbb{I} f$ is a non-split surjection. Since $f$ also does 
not split, $Z:=\operatorname{Im} f$ is a proper submodule of $X$. Let $g \in \operatorname{Hom}_{\Lambda_{1}}(Z, X)$ be an injection. Since $\mathbb{I} g$ is a monomorphism in a torsionfree class $\mathcal{F}_{2}$, it is injective in $\bmod \Lambda_{2}$. Thus $\mathbb{I} f$ factors through a proper submodule $\mathbb{I} Z$ of $\mathbb{I} X$, a contradiction. Hence II restricts to an equivalence $\operatorname{pr} \Lambda_{1} \rightarrow \operatorname{pr} \Lambda_{2}$. Take a progenerator $X \in \operatorname{pr} \Lambda_{1}$ such that $\mathbb{I} X=\Lambda_{2}$. Since $\operatorname{Hom}_{\Lambda_{1}}(X,) \stackrel{\mathbb{I}}{\rightarrow} \operatorname{Hom}_{\Lambda_{2}}(\mathbb{I} X, \mathbb{I}())=\mathbb{I}$ holds on $\mathcal{F}_{1}, \mathbb{I}$ extends to the equivalence $\operatorname{Hom}_{\Lambda_{1}}(X):, \bmod \Lambda_{1} \rightarrow \bmod \Lambda_{2}$, which is easily shown to be the unique extension of $\mathbb{I}$.

1.3. ([I3], [R5]) Let $\mathcal{C}$ be a skeletally small additive category. We denote by $\mathcal{C}(X, Y)$ the set of morphisms from $X$ to $Y$, by $f g$ the composition of $f \in \mathcal{C}(X, Y)$ and $g \in \mathcal{C}(Y, Z)$, by $\mathcal{J}_{\mathcal{C}}$ the Jacobson radical of $\mathcal{C}$, and by ind $\mathcal{C}$ the set of isoclasses of indecomposable objects in $\mathcal{C}$. We call $\mathcal{C}$ Krull-Schmidt if any object is isomorphic to a finite direct sum of objects whose endomorphism rings are local.

Let $\mathcal{C}$ be a Krull-Schmidt category and $\mathbf{A}: X \stackrel{f}{\rightarrow} Y \stackrel{g}{\rightarrow} Z$ a complex. We call $f$ a weak-kernel of $g$ if $\mathcal{C}(, X) \stackrel{\cdot f}{\rightarrow} \mathcal{C}(, Y) \stackrel{\cdot g}{\rightarrow} \mathcal{C}(, Z)$ is exact, and we call $g$ a weak-cokernel of $f$ if $\mathcal{C}(X,) \stackrel{f \cdot}{\leftarrow} \mathcal{C}(Y,) \stackrel{g \cdot}{\leftarrow} \mathcal{C}(Z$, ) is exact. A weak-kernel (resp. weak-cokernel) is called minimal if it has no direct summand of the form $W \rightarrow 0$ (resp. $0 \rightarrow W$ ) with $W \neq 0$ as a complex. Clearly, a minimal weak-(co)kernel is unique up to isomorphism of complexes if it exists. Now we consider the following conditions for $\mathbf{A}$.

(i) $f, g \in \mathcal{J}_{\mathcal{C}}$, and $0 \leftarrow \mathcal{J}_{\mathcal{C}}(X,) \stackrel{f \cdot}{\leftarrow} \mathcal{C}(Y$,$) and \mathcal{C}(, Y) \stackrel{\cdot g}{\rightarrow} \mathcal{J}_{\mathcal{C}}(, Z) \rightarrow 0$ are exact.

(ii) $f$ is a minimal weak-kernel of $g$.

(iii) $g$ is a minimal weak-cokernel of $f$.

We call $\mathbf{A}$ a $\tau$-sequence (resp. right $\tau$-sequence, left $\tau$-sequence) if it satisfies (i)(ii)(iii) (resp. (i)(ii), (i)(iii)). We call a right (resp. left) $\tau$-sequence $\mathbf{A}$ strict if $f$ is a monomorphism (resp. $g$ is an epimorphism) in $\mathcal{C}$. They are analogues of almost split sequences in arbitrary Krull-Schmidt categories.

We call $\mathcal{C}$ a (strict) $\tau$-category if any $X \in \mathcal{C}$ is a right term of some (strict) right $\tau$ sequence and a left term of some (strict) left $\tau$-sequence. Then the right $\tau$-sequence with the right term $X$ (resp. the left $\tau$-sequence with the left term $X$ ) is unique up to isomorphism of complexes, and we denote it by $(X]=\left(\tau^{+} X \stackrel{\nu_{X}^{+}}{\rightarrow} \theta^{+} X \stackrel{\mu_{X}^{+}}{\longrightarrow} X\right)$ (resp. $\left.[X)=\left(X \stackrel{\mu_{X}^{-}}{\longrightarrow} \theta^{-} X \stackrel{\nu_{X}^{-}}{\longrightarrow} \tau^{-} X\right)\right)$. We denote by $\operatorname{ind}_{1}^{+} \mathcal{C}\left(\operatorname{resp} \cdot \operatorname{ind}_{0}^{+} \mathcal{C}, \operatorname{ind}_{1}^{-} \mathcal{C}, \operatorname{ind}_{0}^{-} \mathcal{C}\right)$ the subset of ind $\mathcal{C}$ consisting of $X$ satisfying $\tau^{+} X=0$ (resp. $\theta^{+} X=0, \tau^{-} X=0$, $\left.\theta^{-} X=0\right)$. Up to isomorphism of complexes, $(X]=\left[\tau^{+} X\right)$ and $[Y)=\left(\tau^{-} Y\right]$ hold for any $X \in \operatorname{ind} \mathcal{C}-\operatorname{ind}_{1}^{+} \mathcal{C}$ and $Y \in \operatorname{ind} \mathcal{C}-\operatorname{ind}_{1}^{-} \mathcal{C}$. In particular, $\tau^{+}$and $\tau^{-}$give mutually inverse bijections between ind $\mathcal{C}-\operatorname{ind}_{1}^{+} \mathcal{C}$ and ind $\mathcal{C}-\operatorname{ind}_{1}^{-} \mathcal{C}$ [13, 2.3].

We will use in 5.3 an important property of $\tau$-categories $\mathcal{C}$ which asserts that the factor category $\mathcal{C} /\left[\mathcal{C}^{\prime}\right]$ forms a $\tau$-category again for any subcategories $\mathcal{C}^{\prime}$ of $\mathcal{C}$, where $\left[\mathcal{C}^{\prime}\right]$ is the ideal of $\mathcal{C}$ consisting of morphisms which factor through some object in $\mathcal{C}^{\prime}[$ I4, 1.4].

1.3.1. For a set $Q$, we denote by $\mathbb{Z} Q$ (resp. $\mathbb{N} Q$ ) the free $\mathbb{Z}$-module (resp. free abelian monoid) generated by $Q$. Let $\mathcal{C}$ be a $\tau$-category. We identify $\mathbb{N}$ ind $\mathcal{C}$ with the set of isoclasses of objects in $\mathcal{C}$. We can regard $\theta^{+}, \theta^{-}, \tau^{+}$and $\tau^{-}$as elements of $\operatorname{End}_{\mathbb{Z}}(\mathbb{Z}$ ind $\mathcal{C})$. Put $\phi^{ \pm}:=1_{\mathbb{Z} \text { ind } \mathcal{C}}-\theta^{ \pm}+\tau^{ \pm} \in \operatorname{End}_{\mathbb{Z}}(\mathbb{Z}$ ind $\mathcal{C})$. Let $l: \operatorname{ind} \mathcal{C} \rightarrow \mathbb{N}_{>0}$ be a map. We extend $l$ uniquely to $l \in \operatorname{Hom}_{\mathbb{Z}}(\mathbb{Z}$ ind $\mathcal{C}, \mathbb{Z}$ ). We call $l$ a right (resp. 
left) additive function if $l\left(\phi^{+} X\right) \geq 0$ (resp. $l\left(\phi^{-} X\right) \geq 0$ ) holds for any $X \in \mathcal{C}$ and the equality holds for any $X \in \operatorname{ind} \mathcal{C}-\operatorname{ind}_{1}^{+} \mathcal{C}\left(\right.$ resp. $\left.X \in \operatorname{ind} \mathcal{C}-\operatorname{ind}_{1}^{-} \mathcal{C}\right)$. Then put $l^{+}:=\left\{X \in \operatorname{ind}_{1}^{+} \mathcal{C} \mid l\left(\phi^{+} X\right)>0\right\}\left(\right.$ resp. $\left.l^{-}:=\left\{X \in \operatorname{ind}_{1}^{-} \mathcal{C} \mid l\left(\phi^{-} X\right)>0\right\}\right)$. We call $l$ an additive function if it is left-right additive. Put $l(a):=l(X)-l(Y)$ for $a \in \mathcal{C}(X, Y)$.

1.3.2. Examples. (1) Let $\Lambda$ be an artin algebra and $\mathcal{C}:=\bmod \Lambda$. Then $\mathcal{C}$ forms a strict $\tau$-category with $\operatorname{ind}_{1}^{+} \mathcal{C}=\operatorname{ind}(\operatorname{pr} \Lambda)$ and $\operatorname{ind}_{1}^{-} \mathcal{C}=\operatorname{ind}(\operatorname{in} \Lambda)$. Moreover, $(X]$ gives an almost split sequence for any $X \in \operatorname{ind} \mathcal{C}-\operatorname{ind}_{1}^{+} \mathcal{C}$ [ARS, and $l(X):=$ length $X$ gives an additive function with $l^{ \pm}=\operatorname{ind}_{1}^{ \pm} \mathcal{C}$. More generally, since any contravariantly finite torsionfree class $\mathcal{C}$ over $\Lambda$ has almost split sequences [AS], $\mathcal{C}$ forms a strict $\tau$-category.

(2) Let $\Gamma$ be a semiperfect ring and $\mathcal{C}:=\operatorname{pr} \Gamma$. Then $\mathcal{C}$ forms a strict $\tau$-category if and only if gl.dim $\Gamma \leq 2$ and any simple $\Gamma$ or $\Gamma^{o p}$-module $L$ with $\operatorname{pd} L=2$ satisfies that grade $L=2$ and $\operatorname{Ext}_{\Gamma}^{2}(L, \Gamma)$ is a simple $\Gamma^{o p}$ or $\Gamma$-module. In this case, $\operatorname{ind}_{1}^{+} \mathcal{C}=\{P \in \operatorname{ind} \mathcal{C} \mid \operatorname{pd}$ top $P \leq 1\}$ and $\operatorname{ind}_{1}^{-} \mathcal{C}=\{P \in \operatorname{ind} \mathcal{C} \mid$ pd top $\widehat{P} \leq 1\}$ hold.

(3) Let $\mathcal{Q}$ be a $\tau$-species (=modulated translation quiver in [IT2]) and $\mathcal{C}$ the mesh category of $\mathcal{Q}$ [I3, 8.3 and 8.4]. Then $\mathcal{C}$ is a (not necessarily strict) $\tau$-category. Thus we obtain a bijection between isomorphism classes of $\tau$-species and equivalence classes of completely graded $\tau$-categories [13, 10.1] by taking mesh categories. This structure theorem of completely graded $\tau$-categories was a strong motivation for the introduction of $\tau$-categories in [I3].

1.3.3. [13, 4.1 and 7.2] Let $\mathcal{C}$ be a $\tau$-category. For $X=\sum_{Y \in \text { ind } \mathcal{C}} a_{Y} Y \in \mathbb{Z}$ ind $\mathcal{C}$, put $X_{+}:=\sum_{Y \in \text { ind } \mathcal{C}, a_{Y}>0} a_{Y} Y \in \mathbb{N}$ ind $\mathcal{C}$. Define a map $\theta_{n}^{+}: \mathbb{N}$ ind $\mathcal{C} \rightarrow \mathbb{N}$ ind $\mathcal{C}$ $(n \geq 0)$ by $\theta_{0}^{+}:=1_{\mathbb{N} \text { ind } \mathcal{C}}, \theta_{1}^{+}:=\theta^{+}$and $\theta_{n}^{+} X:=\left(\theta^{+} \theta_{n-1}^{+} X-\tau^{+} \theta_{n-2}^{+} X\right)_{+}$for $n \geq 2$. Then $\theta_{n}^{+}$becomes a monoid monomorphism, and has a functorial meaning such that, for any $X \in \mathcal{C}$, there exists the following commutative diagram such that $\mathcal{C}\left(, \tau^{+} \theta_{n-1}^{+} X\right) \stackrel{\cdot a_{n}}{\longrightarrow} \mathcal{C}\left(, \theta_{n}^{+} X\right) \rightarrow \mathcal{J}_{\mathcal{C}}^{n}(, X) \rightarrow 0$ is exact and $a_{n}$ is in $\mathcal{J}_{\mathcal{C}}$ for any $n \geq 0$ :

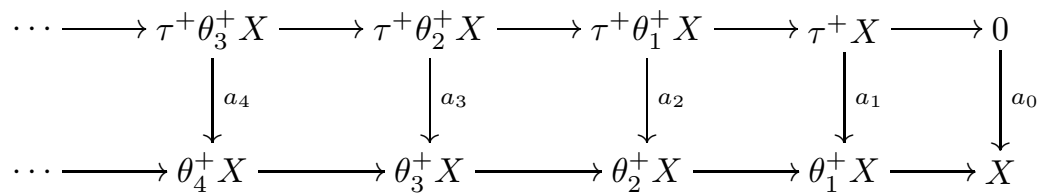

1.4. Let $\mathcal{C}$ be a $\tau$-category and $A, B \in$ ind $\mathcal{C}$. We say that $(A, B)$ is a Nakayama pair if there exists the following commutative diagram for some $n \geq 0$ such that $\left(X_{i} \stackrel{\left(a_{i} g_{i}\right)}{\longrightarrow} Y_{i} \oplus X_{i-1} \stackrel{\left(\begin{array}{c}-f_{i} \\ a_{i-1}\end{array}\right.}{\longrightarrow} Y_{i-1}\right)$ is a $\tau$-sequence for any $i(0<i \leq n)$ :

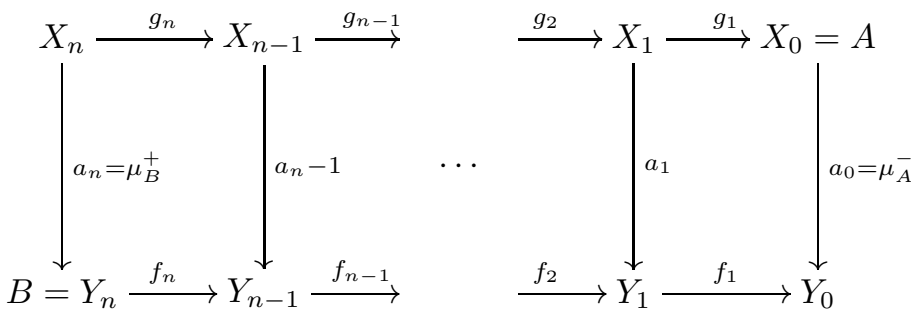


Define $\eta_{i}^{+} \in \operatorname{End}_{\mathbb{Z}}(\mathbb{Z}$ ind $\mathcal{C})(i \geq 0)$ by $\eta_{0}^{+}:=\theta^{-}, \eta_{1}^{+}:=\theta^{+} \circ \theta^{-}-1_{\mathbb{Z} \text { ind } \mathcal{C}}$ and $\eta_{i}^{+}:=\theta^{+} \circ \eta_{i-1}^{+}-\tau^{+} \circ \eta_{i-2}^{+}$for $i \geq 2$. Then $Y_{i}=\eta_{i}^{+} A$ and $X_{i+1}=\tau^{+} \eta_{i}^{+} A$ hold immediately. In particular, $B$ is uniquely determined by $A$, and vice versa. We write $B=\mathrm{n}^{-}(A)$ and $A=\mathrm{n}^{+}(B)$. Note that any right (or left) additive function $l$ satisfies $l\left(a_{0}\right)=l\left(a_{1}\right)=\cdots=l\left(a_{n}\right)$.

1.4.1. Example. (1) Let $\Lambda$ be an order over a complete discrete valuation ring $R$ and let $\mathcal{C}$ be the category of $\Lambda$-lattices [CR]. Then $\mathcal{C}$ forms a $\tau$-category. If $\Lambda$ is representation-finite, then $(A, B)$ is a Nakayama pair for any $B \in \operatorname{ind}(\operatorname{pr} \Lambda)$ and $A:=\operatorname{Hom}_{R}(\widehat{B}, R)$ by [I4, 3.3].

(2) Let $\Lambda$ be a representation-finite artin algebra and $\mathcal{C}:=\bmod \Lambda$. Let $B \in$ $\operatorname{ind}(\operatorname{pr} \Lambda), A:=(\widehat{B})^{*} \in \operatorname{ind}(\operatorname{in} \Lambda)$ and $X:=\operatorname{soc} A=\operatorname{top} B \in \operatorname{ind}(\operatorname{sim} \Lambda)$. In 3.3.1, we will show that $\left(A, \tau^{-} X\right)$ is a Nakayama pair if $A$ is not simple, and $\left(\tau^{+} X, B\right)$ is a Nakayama pair if $B$ is not simple.

1.4.2. We collect basic results on Nakayama pairs, where we refer to [14, 8.1] for (1), [13, 6.4] for (2) and [I4, 2.2] for (3). Let $\mathcal{C}$ be a $\tau$-category.

(1) $(A, B)$ is a Nakayama pair if and only if there exists $n \geq 0$ such that $\eta_{i}^{+} A \in$ $\mathbb{N}\left(\operatorname{ind} \mathcal{C}-\operatorname{ind}_{1}^{+} \mathcal{C}\right)$ for any $i(0 \leq i<n), \eta_{n}^{+} A=B$ and $\eta_{n+1}^{+} A=0$.

(2) Assume $\bigcap_{n \geq 0} \mathcal{J}_{\mathcal{C}}^{n}=0$. If $\mu_{A}^{-}$is not a monomorphism for $A \in \operatorname{ind} \mathcal{C}-\operatorname{ind}_{0}^{-} \mathcal{C}$, then $(A, B)$ is a Nakayama pair for some $B \in \operatorname{ind} \mathcal{C}-\operatorname{ind}_{1}^{+} \mathcal{C}$.

(3) Assume that $\mathcal{C}=\operatorname{pr} \Gamma$ for a semiperfect $\operatorname{ring} \Gamma$. For $A, B \in \operatorname{ind} \mathcal{C}$, let $L:=$ top $A$ be a simple $\Gamma$-module and $M:=\operatorname{top} \widehat{B}$ a simple $\Gamma^{o p}$-module. Then $(A, B)$ is a Nakayama pair if and only if $\operatorname{Tr} L$ has finite length with the socle $M$ and s.grade $(\operatorname{Tr} L) / M \geq 2$ if and only if $\operatorname{Tr} M$ has finite length with the socle $L$ and s.grade $(\operatorname{Tr} M) / L \geq 2$.

1.5. $\tau$-categories were defined "locally" by the properties of simple modules over the category [13]. On the other hand, Rump [R1], [R2] introduced the concept of almost abelian categories, which is given "globally" by the properties of kernels and cokernels. He has shown that they are closely related to tilting theory.

An additive category is called preabelian if any morphism has a kernel and a cokernel. A preabelian category is called almost abelian if kernels are stable under pushout and cokernels are stable under pullback. An almost abelian category is called integral if monomorphisms are stable under pushout and epimorphisms are stable under pullback.

\section{Representation theoretic REALization OF ARTINIAN STRICT $\tau$-CATEGORIES}

2.1. Theorem. Let $\Gamma$ be an artin algebra and $\mathcal{C}:=\operatorname{pr} \Gamma$. Then the following conditions are equivalent.

(1) $\Gamma$ satisfies gl.dim $\Gamma \leq 2$ and the $(2,2)$ and $(2,2)^{o p}$-conditions ( $(1.1)$.

(2) There exists an artin algebra $\Lambda$ and a (faithful) torsion theory $(\mathcal{T}, \mathcal{F}$ ) on $\bmod \Lambda$ such that $\mathcal{C}$ is equivalent to $\mathcal{F}(\S 1.2)$.

$(3) \mathcal{C}$ is a strict $\tau$-category $(\S 1.3)$.

(4) $\mathcal{C}$ is a $\tau$-category with a right additive function (\$1.3.1).

$(5) \mathcal{C}$ is an almost abelian category $(\S 1.5)$.

$(i)^{\text {op }}$ Opposite side version of $(i)(1 \leq i \leq 5)$. 
2.1.1. Remark. (1) The equivalence of $2.1(2)$ and $(2)^{o p}$ follows from the classical cotilting theorem (see 1.2.1(2)), and $(2) \Rightarrow(3)$ follows from the remark in 1.3.2(1).

(2) There exists an artin algebra $\Gamma$ with gl.dim $\Gamma \leq 2$ such that $\Gamma$ satisfies exactly one of the $(2,2)$ and $(2,2)^{o p}$-conditions. For example, such an algebra $\Gamma$ is given by the quiver $\bullet \stackrel{a}{\rightarrow} \bullet \stackrel{b}{\rightarrow} \bullet \leftarrow \bullet$ with the relation $b a=0$.

2.2. Definition. Let $\Gamma$ be an artin algebra and $I^{i}:=I_{\Gamma}^{i}(\Gamma)$. We call a functor $\mathbb{P}: \operatorname{pr} \Gamma \rightarrow \bmod \Lambda$ a (representation theoretic) realization of $\Gamma$ if $\Lambda$ is an artin algebra, $\mathbb{P}$ is full faithful and $\Lambda \in \mathbb{P}(\operatorname{pr} \Gamma)$. This is equivalent to the condition that there exists $Q \in \operatorname{pr} \Gamma$ such that $\Lambda=\operatorname{End}_{\Gamma}(Q), \mathbb{P}$ is isomorphic to $\operatorname{Hom}_{\Gamma}(Q$,$) and$ $I^{0} \oplus I^{1} \in \operatorname{add}(\widehat{Q})^{*}$. We call a realization $\mathbb{P}=\operatorname{Hom}_{\Gamma}(Q$,$) minimal if \operatorname{add}\left(I^{0} \oplus I^{1}\right)=$ $\operatorname{add}(\widehat{Q})^{*}$ holds. A minimal realization of $\Gamma$ is unique up to Morita-equivalence. We sometimes regard $\mathbb{P}=\operatorname{Hom}_{\Gamma}(Q$,$) as a functor \bmod \Gamma \rightarrow \bmod \Lambda$.

2.2.1. Let $\Gamma$ be an artin algebra and $I^{i}:=I_{\Gamma}^{i}(\Gamma)$. For $Q \in \operatorname{pr} \Gamma$, put $\Lambda:=\operatorname{End}_{\Gamma}(Q)$ and $\mathbb{P}:=\operatorname{Hom}_{\Gamma}(Q):, \bmod \Gamma \rightarrow \bmod \Lambda$. Then $\mathbb{P}$ induces an equivalence add $Q \rightarrow$ $\operatorname{pr} \Lambda$, and the conditions (i)-(iv) below are equivalent.

(i) grade $X \geq 2($ resp. grade $X \geq 1)$ holds for any $X \in \bmod \Gamma$ with $\mathbb{P} X=0$.

(ii) $\mathbb{P}$ is full faithful (resp. faithful) on pr $\Gamma$.

(iii) $\mathbb{P}_{X, Y}$ is bijective (resp. injective) for any $X \in \bmod \Gamma$ and $Y \in \operatorname{pr} \Gamma$.

(iv) $I^{0} \oplus I^{1} \in \operatorname{add}(\widehat{Q})^{*}\left(\operatorname{resp} . I^{0} \in \operatorname{add}(\widehat{Q})^{*}\right)$.

Proof. We only prove the assertion for "full faithful". (iii) $\Rightarrow$ (ii) and (i) $\Leftrightarrow$ (iv) are clear (see 3.2(1)).

(ii) $\Rightarrow$ (i) Let $P_{2} \rightarrow P_{1} \rightarrow P_{0} \rightarrow X \rightarrow 0$ be a projective resolution. Then $\mathbb{P} P_{2} \rightarrow$ $\mathbb{P} P_{1} \rightarrow \mathbb{P} P_{0} \rightarrow 0$ is exact. Since $\mathbb{P}$ is full faithful on $\operatorname{pr} \Gamma$, we obtain an exact sequence $\widehat{P}_{2} \leftarrow \widehat{P}_{1} \leftarrow \widehat{P}_{0} \leftarrow 0$ by taking $\operatorname{Hom}_{\Lambda}(, \mathbb{P} \Gamma)$. Thus grade $X \geq 2$ holds.

(i) $\Rightarrow$ (iii) We can take a complex $\mathbf{A}: Q^{m} \stackrel{f_{1}}{\rightarrow} Q^{n} \stackrel{f_{0}}{\rightarrow} X \rightarrow 0$ such that $\Lambda^{m} \stackrel{\mathbb{P} f_{1}}{\longrightarrow}$ $\Lambda^{n} \stackrel{\mathbb{P} f_{0}}{\longrightarrow} \mathbb{P} X \rightarrow 0$ is exact. We obtain an exact sequence $\mathbb{P} \Gamma^{m} \stackrel{\mathbb{P} f_{1}}{\longleftarrow} \mathbb{P} \Gamma^{n} \stackrel{\mathbb{P} f_{0}}{\longleftarrow}$ $\operatorname{Hom}_{\Lambda}(\mathbb{P} X, \mathbb{P} \Gamma) \leftarrow 0$ by taking $\operatorname{Hom}_{\Lambda}(, \mathbb{P} \Gamma)$. On the other hand, put $H_{0}:=\operatorname{Cok} f_{0}$ and $H_{1}:=\operatorname{Ker} f_{0} / \operatorname{Im} f_{1}$. Since $\mathbb{P} H_{j}=0(j=0,1)$ holds, we obtain grade $H_{j} \geq$ 2. Taking $\operatorname{Hom}_{\Gamma}(, \Gamma)$ for $\mathbf{A}$, we obtain an exact sequence $\mathbb{P} \Gamma^{m} \stackrel{\mathbb{P} f_{1} \cdot}{\longleftarrow} \mathbb{P}^{n} \stackrel{\mathbb{P} f_{0}}{\longleftarrow}$ $\operatorname{Hom}_{\Gamma}(X, \Gamma) \leftarrow 0$.

2.2.2. Proof of 2.2. Assume that $Q \in \operatorname{pr} \Gamma$ satisfies $I^{0} \oplus I^{1} \in \operatorname{add}(\widehat{Q})^{*}$ and put $\Lambda:=\operatorname{End}_{\Gamma}(Q)$. Then $\operatorname{Hom}_{\Gamma}(Q$,$) is a realization of \Gamma$ by 2.2.1. Conversely, let $\mathbb{P}$ be a realization of $\Gamma$. Take $Q \in \operatorname{pr} \Gamma$ such that $\mathbb{P} Q=\Lambda$. Since $\operatorname{Hom}_{\Gamma}(Q,) \stackrel{\mathbb{P}}{\rightarrow}$ $\operatorname{Hom}_{\Lambda}(\mathbb{P} Q, \mathbb{P}())=\mathbb{P}$ holds, $\mathbb{P}$ is isomorphic to $\operatorname{Hom}_{\Gamma}(Q$,$) . Moreover, I^{0} \oplus I^{1} \in$ $\operatorname{add}(\widehat{Q})^{*}$ holds by 2.2 .1 .

2.2.3. (1) Let $\Lambda$ be an artin algebra, $\mathcal{C}$ a full subcategory of $\bmod \Lambda$ with an additive generator $M$ and $\Gamma:=\operatorname{End}_{\Lambda}(M)$. Then the functors $\mathbb{Q}:=\operatorname{Hom}_{\Lambda}(M):, \bmod \Lambda \rightarrow$ $\bmod \Gamma$ and $\mathbb{R}:=\operatorname{Hom}_{\Lambda}(, M): \bmod \Lambda \rightarrow \bmod \Gamma^{o p}$ induce equivalences $\mathcal{C} \rightarrow \operatorname{pr} \Gamma$ and $\mathcal{C} \rightarrow \operatorname{pr} \Gamma^{o p}$ such that $\operatorname{Hom}_{\Gamma}(, \Gamma) \circ \mathbb{Q}=\mathbb{R}$ and $\operatorname{Hom}_{\Gamma}(, \Gamma) \circ \mathbb{R}=\mathbb{Q}$ hold on $\mathcal{C}$.

(2) Let $\mathbb{P}: \operatorname{pr} \Gamma \rightarrow \bmod \Lambda$ be a realization of $\Gamma$. Put $\mathcal{C}:=\mathbb{P}(\operatorname{pr} \Gamma)$ and $M:=\mathbb{P} \Gamma$. Then $\mathbb{Q}: \mathcal{C} \rightarrow \operatorname{pr} \Gamma$ in $(1)$ gives a quasi-inverse of $\mathbb{P}: \operatorname{pr} \Gamma \rightarrow \mathcal{C}$. 
2.3. Lemma. Let $\Gamma$ be an artin algebra and $\mathbb{P}: \operatorname{pr} \Gamma \rightarrow \bmod \Lambda$ a realization. Assume that $\Gamma$ satisfies gl.dim $\Gamma \leq 2$ and the $(2,2)^{\text {op }}$-condition. Then $\mathbb{P}(\operatorname{pr} \Gamma)$ is closed under kernels and extensions in $\bmod \Lambda$.

Proof. Take $Q \in \operatorname{pr} \Gamma$ in 2.2 and extend $\mathbb{P}=\operatorname{Hom}_{\Gamma}(Q$,$) to \bmod \Gamma \rightarrow \bmod \Lambda$. Using gl.dim $\Gamma \leq 2$, we can easily show that $\operatorname{pr} \Gamma$ is closed under kernels.

(i) Let $\mathbf{A}: 0 \rightarrow \mathbb{P} P^{\prime} \stackrel{g}{\rightarrow} X \stackrel{f}{\rightarrow} \mathbb{P} P \rightarrow 0$ be an exact sequence in $\bmod \Lambda$ with $P, P^{\prime} \in \operatorname{pr} \Gamma$. We will show that there exists an exact sequence $\mathbf{B}: 0 \rightarrow P^{\prime} \rightarrow L \rightarrow$ $P \rightarrow M \rightarrow 0$ in $\bmod \Gamma$ such that $\mathbf{A}$ is isomorphic to $\mathbb{P} \mathbf{B}$ as a complex.

Take a surjection $d \in \operatorname{Hom}_{\Lambda}\left(\mathbb{P} P_{1}, X\right)$ with $P_{1} \in$ add $Q$ by 2.2.1, and take $a \in$ $\operatorname{Hom}_{\Gamma}\left(P_{1}, P\right)$ such that $d f=\mathbb{P} a$. Taking an exact sequence $\mathbf{C}: 0 \rightarrow \Omega^{2} M \stackrel{b}{\rightarrow} P_{1} \stackrel{a}{\rightarrow}$ $P \rightarrow M \rightarrow 0$, we obtain the following commutative diagram:

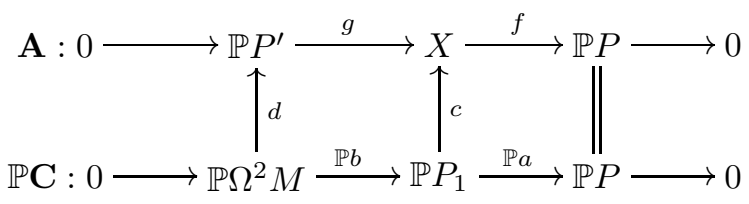

Take $e \in \operatorname{Hom}_{\Gamma}\left(\Omega^{2} M, P^{\prime}\right)$ such that $\mathbb{P} e=d$ by 2.2.1, and define $\mathbf{B}$ by the following push-out diagram:

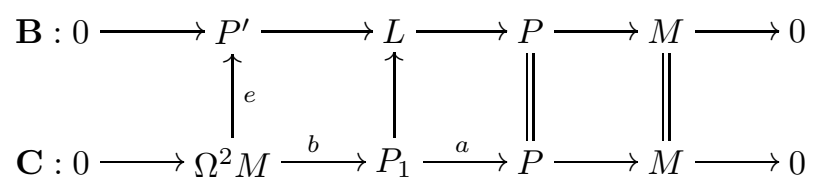

Since both $\mathbf{A}$ and $\mathbb{P B}$ are given by the push-out of $\mathbb{P} \mathbf{C}$ by $d=\mathbb{P} e$, the complexes $\mathbf{A}$ and $\mathbb{P B}$ are isomorphic.

(ii) To show the lemma, take the complex $\mathbf{B}$ in (i). Since grade $M \geq 2$ holds by $\mathbb{P} M=0$, we have an exact sequence $\operatorname{Ext}_{\Gamma}^{2}(M, \Gamma) \leftarrow \widehat{P}^{\prime} \leftarrow \widehat{L} \leftarrow \widehat{P} \leftarrow 0$ by taking $\widehat{()}$. Since $\Gamma$ satisfies the $(2,2)^{o p}$-condition, we obtain the upper exact sequence of the following commutative diagram by taking $\widehat{()}$ again:

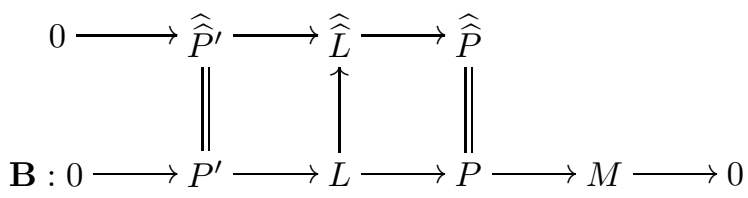

Taking the mapping cone, we obtain an exact sequence $0 \rightarrow L \rightarrow \widehat{\widehat{L}} \rightarrow M$. Thus $X=\mathbb{P} L=\mathbb{P} \widehat{\hat{L}}$ holds. Since gl.dim $\Gamma \leq 2$ holds, we obtain $\widehat{L} \in \operatorname{pr} \Gamma^{o p}$. Thus $X \in \mathbb{P}(\operatorname{pr} \Gamma)$.

2.4. Proof of 2.1. $(1) \Leftrightarrow(1)^{o p}$ is clear, and $(1) \Leftrightarrow(3)$ holds by [I5, 6.3].

$(1) \Rightarrow(2)$ Let $\mathbb{P}=\operatorname{Hom}_{\Gamma}(Q, \quad)$ be a minimal realization of $\Gamma$ which we extend to $\bmod \Gamma \rightarrow \bmod \Lambda$, and let $\mathcal{F}:=\mathbb{P}(\operatorname{pr} \Gamma)$ be a full subcategory of $\bmod \Lambda$. Then $\mathcal{F}$ is closed under extensions by 2.3. We will show that $\mathcal{F}$ is closed under submodules. Fix any $P \in \operatorname{pr} \Gamma$ and an injection $f \in \operatorname{Hom}_{\Lambda}(X, \mathbb{P} P)$. Take a surjection $g \in$ $\operatorname{Hom}_{\Lambda}\left(\mathbb{P} P_{1}, X\right)$ with $P_{1} \in$ add $Q$ by 2.2.1, and take $a \in \operatorname{Hom}_{\Gamma}\left(P_{1}, P\right)$ such that $g f=\mathbb{P} a$. Then $X=\mathbb{P} L$ holds for $L:=\operatorname{Im} a$. The set $\{M \in \bmod \Gamma \mid L \subseteq M \subseteq$ $P, \mathbb{P}(M / L)=0\}$ has a unique maximal element, which we denote by $M$. Then 
$\mathbb{P} M=X$ and $\operatorname{soc}(P / M) \in$ add top $Q=\operatorname{add} \operatorname{soc}\left(I_{\Gamma}^{0}(\Gamma) \oplus I_{\Gamma}^{1}(\Gamma)\right)$ hold. Since $\Gamma$ satisfies the $(2,2)$-condition, an injective hull $I$ of $\operatorname{soc}(P / M)$ satisfies pd $I \leq 1$. Since $I$ gives an injective hull of $P / M$, we obtain $\operatorname{pd} P / M \leq 1$ by gl.dim $\Gamma \leq 2$. Thus $X=\mathbb{P} M$ and $M \in \operatorname{pr} \Gamma$ hold, and $\mathcal{F}$ is a faithful torsionfree class.

$(2) \Rightarrow(4)$ We will show that $l(X):=\operatorname{length}_{\Lambda} X$ gives a right additive function. For any $X \in \mathcal{C},(X]$ gives an exact sequence $0 \rightarrow \tau^{+} X \stackrel{\nu_{X}^{+}}{\rightarrow} \theta^{+} X \stackrel{\mu_{X}^{+}}{\rightarrow} X$ in $\bmod \Lambda$ since the kernel of $\mu_{X}^{+}$in $\bmod \Lambda$ is contained in $\mathcal{F}$ and thus coincides with $\tau^{+} X$. Hence $l\left(\phi^{+} X\right)=l(X)-l\left(\theta^{+} X\right)+l\left(\tau^{+} X\right) \geq 0$ holds. We only have to show that $\mu_{X}^{+}$is surjective for any $X \in \operatorname{ind} \mathcal{C}-\operatorname{ind}_{1}^{+} \mathcal{C}$. Otherwise, the inclusion $Y:=\operatorname{Im} \mu_{X}^{+} \stackrel{a}{\rightarrow} X$ induces an isomorphism $\mathcal{C}(, Y) \stackrel{\cdot a}{\rightarrow} \mathcal{J}_{\mathcal{C}}(, X)$ with $Y \in \mathcal{F}$. Thus $0 \rightarrow Y \stackrel{a}{\rightarrow} X$ gives $(X]$, a contradiction to $X \notin \operatorname{ind}_{1}^{+} \mathcal{C}$.

$(4) \Rightarrow(3)$ Let $l$ be a right additive function. For any $X \in \operatorname{ind} \mathcal{C}-\operatorname{ind}_{1}^{+} \mathcal{C}$, we only have to show that $\nu_{X}^{+}$is a monomorphism. Otherwise, there exists $Y \in \operatorname{ind} \mathcal{C}-$ $\operatorname{ind}_{1}^{+} \mathcal{C}$ such that $\left(\tau^{+} X, Y\right)$ is a Nakayama pair by 1.4.2(2). Then $0>-l(X)=$ $l\left(\nu_{X}^{+}\right)=l\left(\mu_{Y}^{+}\right)=l\left(\tau^{+} Y\right)>0$ holds by 1.4 , a contradiction.

$(2) \Leftrightarrow(5)$ See [R2, Theorem 1].

2.5. We call an artin algebra $\Gamma$ a strict $\tau$-algebra if it satisfies the equivalent conditions in 2.1. We denote by $\operatorname{Gr} \Gamma:=\bigoplus_{n>0} J_{\Gamma}^{n} / J_{\Gamma}^{n+1}$ the associated graded algebra. The Radical Layers Theorem of Igusa-Todorov ([IT1], [BG]), which is one of the most important theorems in the representation theory of algebras, was proved for arbitrary artin algebras and even for $\tau$-categories in [13, 4.2]. Consequently we obtain 2.5.1 below, which implies the following theorem immediately [13, 5.2].

Theorem. Let $\Gamma$ be a strict $\tau$-algebra. Then $\Gamma$ is strongly quasi-Koszul in the sense of Green-Martinez [GM] §5], and $\mathrm{Gr} \Gamma$ is a strict $\tau$-algebra again.

2.5.1. Lemma. Let $\Gamma$ be a strict $\tau$-algebra and $0 \rightarrow P_{2} \stackrel{g}{\rightarrow} P_{1} \stackrel{f}{\rightarrow} P_{0} \rightarrow L \rightarrow 0$ a minimal projective resolution of a simple $\Gamma$-module $L$. Then $0 \rightarrow J_{\Gamma}^{i-1} P_{2} \stackrel{g}{\rightarrow}$ $J_{\Gamma}^{i} P_{1} \stackrel{f}{\rightarrow} J_{\Gamma}^{i+1} P_{0} \rightarrow 0$ is exact for any $i \geq 0$, where we put $J_{\Gamma}^{-1}:=\Gamma$.

\section{Auslander-Regular artin algebra with global Dimension two}

In this section, we study several variations of our Theorem 2.1.

3.1. Theorem. Let $\Gamma$ be an artin algebra and $\mathcal{C}:=\operatorname{pr} \Gamma$. Then the following conditions are equivalent.

(1) $\Gamma$ is an Auslander-regular ring with gl.dim $\Gamma \leq 2(\S 1.1)$.

(2) There exists an artin algebra $\Lambda$ and a (faithful) hereditary torsion theory $(\mathcal{T}, \mathcal{F})$ on $\bmod \Lambda$ such that $\mathcal{C}$ is equivalent to $\mathcal{F}(\S 1.2)$.

$(3) \mathcal{C}$ is a strict $\tau$-category and $\mathrm{n}^{-}$gives a map $\operatorname{ind}_{1}^{-} \mathcal{C}-\operatorname{ind}_{0}^{-} \mathcal{C} \rightarrow \operatorname{ind} \mathcal{C}(\S 1.4)$.

(4) $\mathcal{C}$ is a $\tau$-category with an additive function (\$1.3.1).

(5) $\mathcal{C}$ is an integral almost abelian category $(\S 1.5)$.

$(i)^{o p}$ Opposite side version of $(i)(1 \leq i \leq 5)$.

3.1.1. Lemma. Let $\Lambda$ and $\Gamma$ be artin algebras and $(\mathcal{T}, \mathcal{F})$ a torsion theory on $\bmod \Lambda$. Assume that $\operatorname{pr} \Gamma$ is equivalent to $\mathcal{F}$. Then (1) and (2) below are equivalent, and (3) implies them. If $(\mathcal{T}, \mathcal{F})$ is faithful, then (1)-(3) are equivalent.

(1) $\Gamma$ is an Auslander-regular ring with gl.dim $\Gamma \leq 2$.

(2) If $f \in \operatorname{Hom}_{\Lambda}(Y, X)$ is a surjection with $Y \in \mathcal{F}$ and $X \in \mathcal{T}$, then $f(\operatorname{soc} Y)=0$.

(3) $(\mathcal{T}, \mathcal{F})$ is hereditary. 
Proof. $\Gamma$ satisfies gl.dim $\Gamma \leq 2$ and the $(2,2)$ and $(2,2)^{o p}$-conditions, and $\mathcal{F}$ forms a $\tau$-category by 2.1 . We use the notations in 2.2 .3 and 1.2 (iv).

$(2) \Rightarrow(1)$ To show that $\Gamma$ satisfies the $(1,1)^{o p}$-condition, we will show grade $M>0$ for any simple $\Gamma$-module $L$ and a submodule $M$ of $\operatorname{Ext}_{\Gamma}^{1}(L, \Gamma)$. Since $\operatorname{pd} L=2 \mathrm{im}$ plies grade $L=2$ by 1.3.2(2), we can assume $\operatorname{pd} L=1$. Take projective resolutions $0 \rightarrow \mathbb{Q} Y \stackrel{\mathbb{Q} f}{\longrightarrow} \mathbb{Q} X \rightarrow L \rightarrow 0$ and $0 \leftarrow M \stackrel{a}{\leftarrow} \mathbb{R} W$. Then $0 \leftarrow \operatorname{Ext}_{\Gamma}^{1}(L, \Gamma) \leftarrow$ $\mathbb{R} Y \stackrel{\mathbb{R} f}{\longleftarrow} \mathbb{R} X$ is exact, and $a$ lifts to $\mathbb{R} d \in \operatorname{Hom}_{\Gamma}(\mathbb{R} W, \mathbb{R} Y)$. Take an exact sequence $Y \stackrel{(f d)}{\longrightarrow} X \oplus W \stackrel{\left(\begin{array}{l}g \\ e\end{array}\right)}{\longrightarrow} V \rightarrow 0$. Then $0 \leftarrow M \stackrel{a}{\longleftarrow} \mathbb{R} W \stackrel{\mathbb{R}\left(e \alpha_{V}\right)}{\longleftarrow} \mathbb{R} \circ \mathbb{F} V$ gives a projective resolution for $e \alpha_{V} \in \operatorname{Hom}_{\Lambda}(W, \mathbb{F} V)$. Since $0 \rightarrow \widehat{M} \rightarrow \mathbb{Q} W \stackrel{\mathbb{Q}\left(e \alpha_{V}\right)}{\longrightarrow} \mathbb{Q} \circ \mathbb{F} V$ is exact, we only have to show that $e \alpha_{V}$ is injective. Put $U:=\left(\begin{array}{l}g \\ e\end{array}\right)^{-1}(\mathbb{T} V) \subseteq X \oplus W$. Then $\left(\begin{array}{l}g \\ e\end{array}\right)(\operatorname{soc} U)=0$ holds by (2). Since $f$ and $e$ are injective, $W \cap$ soc $U=0$ holds. Thus $W \cap U=0$ holds, and we obtain the assertion.

(1) $\Rightarrow(2)$ Let $0 \rightarrow Z \stackrel{g}{\rightarrow} Y \stackrel{f}{\rightarrow} X \rightarrow 0$ be an exact sequence such that $Y \in \mathcal{F}$, $X \in \mathcal{T}$ and soc $Y \nsubseteq Z$. Then there exists an injection $\left(\begin{array}{l}g \\ h\end{array}\right) \in \operatorname{Hom}_{\Lambda}(Z \oplus W, Y)$ with $W \neq 0$. Define $L \in \bmod \Gamma$ by an exact sequence $0 \rightarrow \mathbb{Q}(Z \oplus W) \stackrel{\mathbb{Q}\left(\begin{array}{l}g \\ h\end{array}\right)}{\longrightarrow} Y \rightarrow L \rightarrow 0$. Then $0 \leftarrow \operatorname{Ext}_{\Gamma}^{1}(L, \Gamma) \stackrel{a}{\leftarrow} \mathbb{R}(Z \oplus W) \stackrel{\mathbb{R}\left(\begin{array}{l}g \\ h\end{array}\right)}{\longleftarrow} \mathbb{R} Y$ is exact. Since $\mathbb{R} g$ is injective, $a$ restricts to an injection $\mathbb{R} W \rightarrow \operatorname{Ext}_{\Gamma}^{1}(L, \Gamma)$. Thus $\mathbb{Q} W=\operatorname{Hom}_{\Gamma}(\mathbb{R} W, \Gamma)=0$, a contradiction.

$(3) \Rightarrow(2)$ For any simple submodule $Z$ of $Y, Z \in \mathcal{F}$ and $f(Z) \in \mathcal{T}$ imply $f(Z)=0$.

$(2)+(\Lambda \in \mathcal{F}) \Rightarrow(3)$ Let $X$ be a submodule of $Y \in \mathcal{T}$ and let $f \in \operatorname{Hom}_{\Lambda}(\mathbb{F} X, Y / \mathbb{T} X)$ be a natural injection. Take a surjection $\left(\begin{array}{l}g \\ f\end{array}\right) \in \operatorname{Hom}_{\Lambda}(P \oplus \mathbb{F} X, Y / \mathbb{T} X)$ with $P \in$ $\operatorname{pr} \Lambda$. By $P \oplus \mathbb{F} X \in \mathcal{F}$ and $Y / \mathbb{T} X \in \mathcal{T}$, we obtain $f(\operatorname{soc} \mathbb{F} X)=0$. Thus soc $\mathbb{F} X=0$ and $X \in \mathcal{T}$.

\subsubsection{Proof of 3.1. (4) $\Leftrightarrow(4)^{o p}$ holds clearly.}

$(3) \Rightarrow(1)$ We use the notation in 2.2.3. To show that $\Gamma$ satisfies the $(1,1)^{o p_{-}}$ condition, we will show s.grade $\operatorname{Ext}_{\Gamma}^{1}(L, \Gamma) \geq 1$ for any simple $\Gamma$-module $L$ with $\operatorname{pd} L=1$. Put $L=\operatorname{top} \mathbb{R} A$ for $A \in \operatorname{ind}_{1}^{-} \mathcal{C}-\operatorname{ind}_{0}^{-} \mathcal{C}, B:=\mathrm{n}^{-}(A) \in \operatorname{ind} \mathcal{C}$ and $M:=\operatorname{top} \mathbb{Q} B$. Then we have an exact sequence $0 \rightarrow M \rightarrow \operatorname{Ext}_{\Gamma}^{1}(L, \Gamma) \rightarrow N \rightarrow$ 0 , where s.grade $N \geq 2$ holds by 1.4.2(3). Since $\operatorname{grade} \operatorname{Ext}_{\Gamma}^{1}(L, \Gamma) \geq 1$ holds by pd $L=1$, we obtain s.grade $M=\operatorname{grade} M \geq 1$ by taking $\operatorname{Hom}_{\Gamma}(, \Gamma)$. Thus s.grade $\operatorname{Ext}_{\Gamma}^{1}(L, \Gamma) \geq 1$ holds.

$(1) \Rightarrow(2)$ Immediate from 2.1 and 3.1.1.

(2) $\Rightarrow(4)$ By 1.2, we can put $\mathcal{F}=\mathbf{S}^{\perp}$ and $\mathcal{T}={ }^{\perp} \mathcal{F}$ for $\mathbf{S} \subseteq \operatorname{ind}(\operatorname{sim} \Lambda)$. For $X \in \bmod \Lambda$, we denote by $l(X)$ the number of its composition factors which is not in $\mathbf{S}$. We will show that $l$ gives an additive function. By the argument in the proof of $2.1(2) \Rightarrow(4), l$ is right additive. For any $X \in \operatorname{ind}_{1}^{-} \mathcal{C}$, take an exact sequence $X \stackrel{\mu_{X}^{-}}{\rightarrow} \theta^{-} X \rightarrow Y \rightarrow 0$ in $\bmod \Lambda$. Since $\mu_{X}^{-}$is an epimorphism in $\mathcal{C}$, we obtain $Y \in \mathcal{T}$ and $l(Y)=0$. Thus $l(X) \geq l\left(\theta^{-} X\right)$ holds for any $X \in \operatorname{ind}_{1}^{-} \mathcal{C}$, and $l$ is additive.

$(4) \Rightarrow(3) \mathcal{C}$ is strict by 2.1 . Let $l$ be an additive function. In the proof below, we have to use concepts in [I3]. Take $X \in \operatorname{ind}_{1}^{-} \mathcal{C}-\operatorname{ind}_{0}^{-} \mathcal{C}$ and let $\mathbf{a}=\left(a_{i}\right)_{0 \leq i}$ be the right ladder of $\mu_{X}^{-}$for $a_{i} \in \mathcal{C}\left(X_{i}, Y_{i}\right)$. If $\mathbf{a}$ is not essential, then $(X, Y)$ is a Nakayama pair for some $Y \in \operatorname{ind} \mathcal{C}-\operatorname{ind}_{1}^{+} \mathcal{C}$ by [13, 6.4]. Thus we assume that a is essential. Take a maximal number $n$ such that $Y_{n} \neq 0$. Then $Y_{n} \in \operatorname{add}\left(\operatorname{ind}_{1}^{+} \mathcal{C}\right)$ 
and $a_{n}=\mu_{Y_{n}}^{+}$hold. Let $\mathbf{c}=\left(c_{i}\right)_{0 \leq i \leq n}$ be the left ladder of $a_{n}$ for $c_{i} \in \mathcal{C}\left(A_{i}, B_{i}\right)$. Since $A_{i}$ has no direct summands in $\operatorname{ind}_{1}^{-} \mathcal{C}$ for any $i(0 \leq i<n)$ by [I3, 6.3.1(1)], c is invertible by [13, 6.2.1]. Since $l\left(c_{i}\right)=l\left(a_{n}\right) \leq 0$ holds, $B_{i}=0$ implies $A_{i}=0$. Hence $A_{i} \neq 0$ holds for any $i(0 \leq i \leq n)$ inductively. Since $\left(a_{i}\right)_{0 \leq i \leq n}$ is invertible by [13, $6.3 .1(2)(\mathrm{i})],\left(X, Y_{n}\right)$ is a Nakayama pair and $\mathrm{n}^{-}(X)=Y_{n}$.

$(2) \Rightarrow(5)$ follows from [R2 Lemma 6], and $(5) \Rightarrow(1)$ follows from a quite similar argument as in the proof of $3.1 .1(2) \Rightarrow(1)$.

3.2. Let $\Gamma$ be an artin algebra with $I^{i}:=I_{\Gamma}^{i}(\Gamma)$.

(1) The bijection soc : ind (in $\Gamma) \rightarrow$ ind $(\operatorname{sim} \Gamma)$ induces the maps below. The first and second maps are bijective, and so is the third map if gl.dim $\Gamma<\infty$ :

$$
\begin{aligned}
\operatorname{ind}\left(\operatorname{add} I^{n}\right) & \rightarrow\left\{L \in \operatorname{ind}(\operatorname{sim} \Gamma) \mid \operatorname{Ext}_{\Gamma}^{n}(L, \Gamma) \neq 0\right\} \\
\operatorname{ind}\left(\operatorname{add} I^{n}\right)-\bigcup_{i<n} \operatorname{ind}\left(\operatorname{add} I^{i}\right) & \rightarrow\{L \in \operatorname{ind}(\operatorname{sim} \Gamma) \mid \operatorname{grade} L=n\} \\
\operatorname{ind}\left(\operatorname{add} I^{n}\right)-\bigcup_{i>n} \operatorname{ind}\left(\operatorname{add} I^{i}\right) & \rightarrow\{L \in \operatorname{ind}(\operatorname{sim} \Gamma) \mid \operatorname{pd} L=n\}
\end{aligned}
$$

(2) Assume that the conditions in 2.1 are satisfied and $(\mathcal{T}, \mathcal{F})$ is faithful. Let $\mathbb{P}$ : $\operatorname{pr} \Gamma=\mathcal{C} \stackrel{\sim}{\rightarrow} \mathcal{F} \subset \bmod \Lambda$ be the composition. Then $\operatorname{ind}\left(\operatorname{add}\left(I^{0} \oplus I^{1}\right)\right) \cap \operatorname{ind}\left(\operatorname{add} I^{2}\right)=$ $\emptyset$ holds, and $\mathbb{P}$ is a minimal realization of $\Gamma$ given by $\mathbb{P}=\operatorname{Hom}_{\Gamma}(Q$, ) for some $Q \in \operatorname{pr} \Gamma$. We have the bijections below, where the middle map is given by the projective cover:

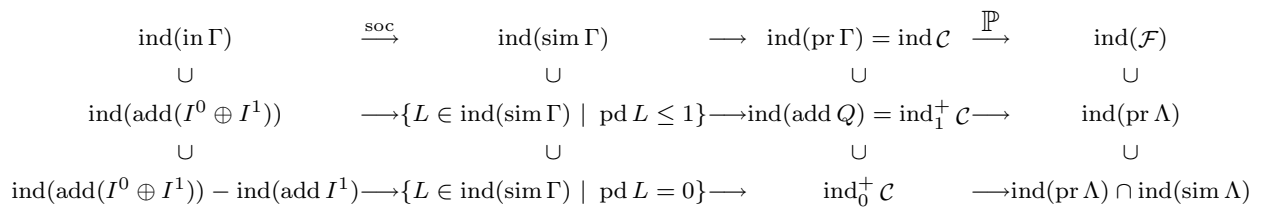

(3) In (2), assume that the conditions in 3.1 are satisfied and $l$ is an additive function of $\mathcal{C}$. Then the bijections in (2) induce the bijections (i), and the equalities (ii) hold:

$$
\begin{aligned}
& \operatorname{ind}\left(\operatorname{add} I^{0}\right) \longrightarrow\{L \in \operatorname{ind}(\operatorname{sim} \Gamma) \mid \operatorname{grade} L=0\} \longrightarrow l^{+} \longrightarrow\{X \in \operatorname{ind}(\operatorname{pr} \Lambda) \mid \operatorname{top} X \in \mathcal{F}\} \\
& \qquad \begin{array}{c}
l^{+}=\operatorname{ind}_{0}^{+} \mathcal{C} \cup\left\{X \in \operatorname{ind}_{1}^{+} \mathcal{C}-\operatorname{ind}_{0}^{+} \mathcal{C} \mid \mathrm{n}^{+}(X) \in \operatorname{ind} \mathcal{C}-\operatorname{ind}_{1}^{+} \mathcal{C}\right\} \\
=\left\{X \in \operatorname{ind}_{1}^{+} \mathcal{C} \mid \mu_{X}^{+} \text {is not an epimorphism }\right\}
\end{array}
\end{aligned}
$$

Proof. (1) Taking $\operatorname{Hom}_{\Gamma}(L, \quad)$ for a minimal injective resolution $0 \rightarrow \Gamma \rightarrow I^{0} \rightarrow$ $I^{1} \rightarrow \cdots$, we obtain the first bijection, which implies others.

(2) The former assertion follows from 1.3.2(2), and soc induces the left bijections by (1). Since $\mathbb{P}$ is a realization by $\Lambda \in \mathbb{P}(\operatorname{pr} \Gamma)$, we can take $Q \in \operatorname{pr} \Gamma$ such that $\mathbb{P}=\operatorname{Hom}_{\Gamma}(Q, \quad)$ and $I^{0} \oplus I^{1} \in \operatorname{add}(\widehat{Q})^{*}$ by 2.2 . To show that $\mathbb{P}$ is minimal, fix $P \in \operatorname{ind}(\operatorname{pr} \Gamma)$. Then $P \in \operatorname{ind}_{1}^{+} \mathcal{C} \Leftrightarrow \operatorname{pd}$ top $P \leq 1 \Leftrightarrow(\widehat{P})^{*} \in \operatorname{add}\left(I^{0} \oplus I^{1}\right) \Rightarrow P \in$ add $Q \Leftrightarrow \mathbb{P} P \in \operatorname{pr} \Lambda(*)$ holds. Assume $\mathbb{P} P \in \operatorname{pr} \Lambda$, and take $f \in \mathcal{C}\left(P^{\prime}, P\right)$ such that $\mathbb{P} f$ gives the inclusion $J_{\Lambda} \mathbb{P} P \subset \mathbb{P} P$. Since $(P]=\left(0 \rightarrow P^{\prime} \stackrel{f}{\rightarrow} P\right)$ holds, we obtain $P \in \operatorname{ind}_{1}^{+} \mathcal{C}$. Thus the above five conditions in $(*)$ are equivalent. Consequently, $\mathbb{P}$ is minimal by $\operatorname{add}\left(I^{0} \oplus I^{1}\right)=\operatorname{add}(\widehat{Q})^{*}$, and we obtain the desired bijections.

(3) Clearly, $l^{+} \supseteq \operatorname{ind}_{0}^{+} \mathcal{C}$ holds. Let $X \in \operatorname{ind}_{1}^{+} \mathcal{C}-\operatorname{ind}_{0}^{+} \mathcal{C}$ and $Y:=\mathrm{n}^{+}(X) \in \operatorname{ind} \mathcal{C}$. Then $0 \geq l\left(\mu_{X}^{+}\right)=l\left(\mu_{Y}^{-}\right)$holds by 1.4. Since $l\left(\mu_{Y}^{-}\right) \geq 0$ if and only if $Y \in \operatorname{ind}_{1}^{-} \mathcal{C}$, we obtain the first equality in (ii). The second equality in (ii) follows from [13. 6.4.1(2)]. 
Take $I \in \operatorname{ind}\left(\operatorname{add}\left(I^{0} \oplus I^{1}\right)\right)$. Let $L:=\operatorname{soc} I$, let $0 \rightarrow P_{1} \stackrel{f}{\rightarrow} P_{0} \rightarrow L \rightarrow 0$ be a minimal projective resolution and let $X:=\mathbb{P} P_{0} \in \mathcal{F}$. Then $\left(P_{0}\right]=\left(0 \rightarrow P_{1} \stackrel{f}{\rightarrow} P_{0}\right)$ holds for $\operatorname{pr} \Gamma=\mathcal{C}$. Moreover, $I \in$ add $I^{0}$ if and only if grade $L=0$ if and only if $f$ is not an epimorphism in $\mathcal{C}$ if and only if $P_{0} \in l^{+}$by (ii). Since $\mathbb{P} L$ is a simple $\Lambda$-module, we obtain an exact sequence $0 \rightarrow \mathbb{P} P_{1} \stackrel{\mathbb{P} f}{\longrightarrow} X \rightarrow \operatorname{top} X \rightarrow 0$ in $\bmod \Lambda$ by taking $\mathbb{P}$. Hence $f$ is not an epimorphism in $\mathcal{C}$ if and only if $\mathbb{P} f$ is also not an epimorphism in $\mathcal{F}$ if and only if top $X \in \mathcal{F}$. Thus (i) holds.

3.3. Now we obtain the following theorem which implies the classical theorem of Auslander in 0.2. Recall that we call an artin algebra $\Gamma$ an Auslander algebra if gl.dim $\Gamma \leq 2$ and $\Gamma$ satisfies the $(1,2)$-condition, namely $\operatorname{dom}$.dim $\Gamma \geq 2$. Notice that the equivalence of (2) and (3) below is a special case of [R3, Cor. of Prop. 6].

Theorem. Let $\Gamma$ be an artin algebra and $\mathcal{C}:=\operatorname{pr} \Gamma$. Then the following conditions are equivalent.

(1) $\Gamma$ is an Auslander algebra.

(2) $\Gamma$ is an Auslander-regular ring with gl.dim $\Gamma \leq 2$, and any simple $\Gamma$-module $L$ with $\operatorname{pd} L=1$ satisfies grade $L=0$.

(3) There exists an artin algebra $\Lambda$ such that $\mathcal{C}$ is equivalent to $\bmod \Lambda$.

(4) $\mathcal{C}$ is a strict $\tau$-category and $\mathrm{n}^{-}$gives a map $\operatorname{ind}_{1}^{-} \mathcal{C}-\operatorname{ind}_{0}^{-} \mathcal{C} \rightarrow \operatorname{ind} \mathcal{C}-\operatorname{ind}_{1}^{+} \mathcal{C}$.

$(5) \mathcal{C}$ is a $\tau$-category with an additive function $l$ such that $l^{-}=\operatorname{ind}_{1}^{-} \mathcal{C}$.

(6) $\mathcal{C}$ is an abelian category.

$(i)^{\text {op }}$ Opposite side version of $(i)(1 \leq i \leq 6)$.

Proof. Each of the above conditions implies that $\Gamma$ is Auslander-regular with gl.dim $\Gamma \leq 2$ by 3.1. Obviously (1) is equivalent to $\operatorname{add}\left(I^{0} \oplus I^{1}\right)=\operatorname{add} I^{0}$. Now $3.2(3)$ immediately implies $(1) \Leftrightarrow(2) \Leftrightarrow(3) \Leftrightarrow(4)^{o p} \Leftrightarrow(5)^{o p}$ since $\{X \in \operatorname{ind}(\operatorname{pr} \Lambda)$ top $X \in F\}=\operatorname{ind}(\operatorname{pr} \Lambda)$ is equivalent to $F=\bmod \Lambda$. Since $(\bmod \Lambda)^{o p}$ is equivalent to $\bmod \Lambda^{o p}$, we obtain $(3) \Leftrightarrow(3)^{o p}$, and $(3) \Rightarrow(6)$ is obvious. We will show $(6) \Rightarrow(2)$. We only have to show the latter assertion. Let $0 \rightarrow P_{1} \stackrel{f}{\rightarrow} P_{0} \rightarrow L \rightarrow 0$ be a minimal projective resolution. Since $f$ is a non-invertible monomorphism in an abelian category $\mathcal{C}, f$ is not an epimorphism in $\mathcal{C}$. Thus $\widehat{f}: \widehat{P}_{0} \rightarrow \widehat{P}_{1}$ is not a monomorphism, and $\widehat{L} \neq 0$ holds.

3.3.1. Corollary. Let $\Lambda$ be a representation-finite artin algebra and $\mathcal{C}:=\bmod \Lambda$. Let $B \in \operatorname{ind}(\operatorname{pr} \Lambda), A:=(\widehat{B})^{*} \in \operatorname{ind}(\operatorname{in} \Lambda)$ and $X:=\operatorname{soc} A=\operatorname{top} B \in \operatorname{ind}(\operatorname{sim} \Lambda)$. Then $\left(A, \tau^{-} X\right)$ is a Nakayama pair if $A$ is not simple, and $\left(\tau^{+} X, B\right)$ is a Nakayama pair if $B$ is not simple.

Proof. Since $\mathrm{n}^{-}(A) \in \operatorname{ind}(\bmod \Lambda)-\operatorname{ind}(\operatorname{pr} \Lambda)$ holds by $3.3(4)$, there exists an exact sequence $0 \rightarrow \tau^{+} \mathrm{n}^{-}(A) \rightarrow A \stackrel{\mu_{A}^{-}}{\rightarrow} \theta^{-} A \rightarrow 0$ by the definition 1.4. Since $\mu_{A}^{-}$is a natural surjection $A \rightarrow A / X$, we obtain $\tau^{+} \mathrm{n}^{-}(A)=X$. Thus $\left(A, \tau^{-} X\right)$ is a Nakayama pair.

3.4. We denote by $\bmod { }_{s p} \Lambda$ the full subcategory of $\bmod \Lambda$ consisting of $\Lambda$-modules whose socles are projective. Such categories $\bmod _{s p} \Lambda$ play an important role in representation theory. They are closely related to the representation theory of partially ordered sets, vector space categories, and orders over complete discrete valuation rings (see $[\mathrm{S}]$ ). The theorem below asserts that the endomorphism ring of $\bmod _{s p} \Lambda$ is characterized in terms of a diagonal Auslander-regular ring, where 
we call an Auslander-regular ring $\Gamma$ diagonal if any non-zero direct summand $I$ of $I_{\Gamma}^{i}(\Gamma)$ satisfies $\operatorname{fd}_{\Gamma} I=i$.

Theorem. Let $\Gamma$ be an artin algebra and $\mathcal{C}:=\operatorname{pr} \Gamma$. Then the following conditions are equivalent.

(1) $\Gamma$ is a diagonal Auslander-regular ring with gl.dim $\Gamma \leq 2$.

(2) $\Gamma$ is an Auslander-regular ring with $\operatorname{gl} \cdot \operatorname{dim} \Gamma \leq 2$, and any simple $\Gamma$-module $L$ with $\operatorname{pd} L=1$ satisfies grade $L=1$.

(3) There exists an artin algebra $\Lambda$ such that $\mathcal{C}$ is equivalent to $\bmod { }_{s p} \Lambda$.

(4) $\mathcal{C}$ is a strict $\tau$-category and $\mathrm{n}^{-}$gives a (bijective) map $\operatorname{ind}_{1}^{-} \mathcal{C}-\operatorname{ind}_{0}^{-} \mathcal{C} \rightarrow$ $\operatorname{ind}_{1}^{+} \mathcal{C}-\operatorname{ind}_{0}^{+} \mathcal{C}$.

$(5) \mathcal{C}$ is a $\tau$-category with an additive function $l$ such that $l^{-}=\operatorname{ind}_{0}^{-} \mathcal{C}$.

$(i)^{o p}$ Opposite side version of $(i)(1 \leq i \leq 5)$.

3.4.1. Let $\Gamma$ be a 1-Gorenstein artin algebra. Then any simple $\Gamma$-module $L$ with grade $L=0$ is projective if and only if any simple $\Gamma^{o p}$-module $L$ with grade $L=0$ is projective.

Proof. We will show the "only if" part. Assume that a simple $\Gamma^{o p}$-module $L$ satisfies grade $L=0$ and $\operatorname{pd} L>0$. Take a projective resolution $0 \rightarrow \Omega L \stackrel{g}{\rightarrow} P_{0} \stackrel{f}{\rightarrow} L \rightarrow 0$. We have an exact sequence $0 \leftarrow \operatorname{Ext}_{\Gamma}^{1}(L, \Gamma) \stackrel{a}{\leftarrow} \widehat{\Omega} L \stackrel{\widehat{g}}{\leftarrow} \widehat{P}_{0} \stackrel{\widehat{f}}{\leftarrow} \widehat{L} \leftarrow 0$. Then the injective hull $b \in \operatorname{Hom}_{\Gamma}\left(\widehat{P}_{0}, I\right)$ satisfies $I \in \operatorname{pr} \Gamma$ by the $(1,1)$-condition. Suppose that $b$ is not an isomorphism. Since $\widehat{b}$ factors through $g$, it follows that $b$ factors through $\widehat{g}$. Thus $\widehat{L}=0$, a contradiction. Hence $\widehat{P}_{0} \in \operatorname{ind}($ in $\Lambda)$ holds. Since $\widehat{\Omega} L \neq 0$ by pd $L>0$, we can take an injection $c \in \operatorname{Hom}_{\Gamma}(M, \widehat{\Omega} L)$ for a simple $\Gamma$-module $M$. Then $M \in \operatorname{pr} \Gamma$ holds by grade $M=0$. Since $c a=0$ holds by the $(1,1)$-condition, there exists $c^{\prime}$ such that $c=c^{\prime} \widehat{g}$. Since soc $\widehat{P}_{0}$ is simple, $c^{\prime}$ factors through $\widehat{f}$. Thus $c=0$, a contradiction.

3.4.2. Proof of 3.4. $(2) \Leftrightarrow(2)^{o p}$ holds by 3.4.1. The diagonal condition is equivalent to ind $\left(\operatorname{add}\left(I^{0} \oplus I^{1}\right)\right)-\operatorname{ind}\left(\operatorname{add} I^{1}\right)=\operatorname{ind}\left(\operatorname{add} I^{0}\right)$. Now a similar argument as in the proof of 3.3 works to show $(1) \Leftrightarrow(2) \Leftrightarrow(3) \Leftrightarrow(4)^{o p} \Leftrightarrow(5)^{o p}$ since $\{X \in$ $\operatorname{ind}(\operatorname{pr} \Lambda) \mid \operatorname{top} X \in F\}=\operatorname{ind}(\operatorname{pr} \Lambda) \cap \operatorname{ind}(\operatorname{sim} \Lambda)$ is equivalent to $F=\bmod _{s p} \Lambda$.

3.5. We obtain the following corollary by 1.2.1, where the case $i=2$ is Auslander's correspondence 0.2 .

Corollary. There exists a bijection between (1-i) and (2-i) below $(1 \leq i \leq 4)$, which is given by $\mathcal{C} \mapsto \Gamma:=\mathcal{C}(M, M)$.

(1) Equivalence classes of additive categories $\mathcal{C}$ with additive generators $M$ such that

$(1-1) \mathcal{C}$ is a faithful torsionfree class over an artin algebra,

$(1-2) \mathcal{C}$ is a faithful hereditary torsionfree class over an artin algebra,

$(1-3) \mathcal{C}=\bmod \Lambda$ over an artin algebra $\Lambda$,

$(1-4) \mathcal{C}=\bmod _{s p} \Lambda$ over an artin algebra $\Lambda$.

(2) Morita-equivalence classes of artin algebras $\Gamma$ such that

$(2-1) \Gamma$ satisfies $\operatorname{gl} \cdot \operatorname{dim} \Gamma \leq 2$ and the $(2,2)$ and $(2,2)^{o p}$-conditions,

(2-2) $\Gamma$ is an Auslander-regular ring with gl.dim $\Gamma \leq 2$,

(2-3) $\Gamma$ is an Auslander algebra,

(2-4) $\Gamma$ is a diagonal Auslander-regular ring with gl.dim $\Gamma \leq 2$. 
3.6. In this section, we collect some homological results and questions.

3.6.1. Symmetry. We have obtained a few left-right symmetries in previous sections. Moreover, recall that $\Gamma$ is $n$-Gorenstein if and only if $\Gamma^{o p}$ is also by [FGR, 3.7], and dom.dim $\Gamma=$ dom.dim $\Gamma^{o p}$ holds by $[\mathrm{H} 2$. These left-right symmetries are generalized as follows: We say that $l \geq 0$ is a dominant number of $\Gamma$ if $\mathrm{fd} I_{\Gamma}^{i}(\Gamma)<\mathrm{fd} I_{\Gamma}^{l}(\Gamma)$ holds for any $i(0 \leq i<l)$.

Theorem ([9] 1.1, 2.4]). Let $l$ and $n$ be positive integers.

(1) For an $n$-Gorenstein ring $\Gamma$, the set of dominant numbers of $\Gamma$ smaller than $n$ coincides with that of $\Gamma^{o p}$. Any dominant number $l$ of $\Gamma$ with $l<n$ satisfies fd $I_{\Gamma}^{l}(\Gamma)=l$.

(2) Assume that a noetherian ring $\Gamma$ satisfies the $(l, l)$ and $(l, l)^{o p}$-conditions. Then $\Gamma$ satisfies the $(l, n)$-condition if and only if it satisfies the $(l, n)^{o p}$-condition.

3.6.2. Question. Let $\Gamma$ be an artin algebra. Is the condition that $\Gamma$ is diagonal Auslander-regular left-right symmetric? This is true if gl.dim $\Gamma \leq 2$ by 3.4. More generally, is the following condition $(*)$ left-right symmetric for an $n$-Gorenstein ring $\Gamma$ ? This is true for $n=1$ by 3.4.1.

(*) Any simple $\Gamma$-module $L$ with grade $L=i$ satisfies $\operatorname{pd} L=i$ for any $i(0 \leq$ $i<n)$.

3.6.3. Duality. Let $\Gamma$ be a noetherian ring, $\mathbb{E}_{n}:=\operatorname{Ext}_{\Gamma}^{n}(, \Gamma)$ and $\mathbb{F}_{n}:=\operatorname{soc} \mathbb{E}_{n}$. Consider the following condition $\left(\mathrm{D}_{n}\right)$.

$\left(\mathrm{D}_{n}\right) \mathbb{F}_{n}$ gives a bijection between isoclasses of simple $\Gamma$-modules $L$ with grade $L$ $=n$ and that of $\Gamma^{o p}$. Moreover, $\mathbb{F}_{n} \circ \mathbb{F}_{n} L$ is isomorphic to $L$, and s.grade $\mathbb{E}_{n} L / \mathbb{F}_{n} L>$ $n$ holds.

If an artin algebra $\Gamma$ satisfies gl.dim $\Gamma \leq 2$ and the $(2,2)$ and $(2,2)^{o p}$-conditions, then $\left(\mathrm{D}_{2}\right)$ holds by 2.1 and $1.3 .2(2)$. Moreover, if $\Gamma$ is Auslander-regular, then 3.1 and 1.4.2(3) imply that $\left(\mathrm{D}_{1}\right)$ holds, and more strongly $\mathbb{F}_{1}$ gives an injection from isoclasses of simple $\Gamma$-modules $L$ with grade $L \leq 1$ to isoclasses of simple $\Gamma^{o p}$-modules. These observations are generalized as follows:

Theorem. Let $n \geq 0$ and let $\Gamma$ be a noetherian algebra satisfying the $(l, l)$ and $(l, l)^{o p}$-conditions for $l=n, n+1$. Then $\left(\mathrm{D}_{n}\right)$ holds. Moreover, if gl.dim $\Gamma=n \geq 2$, then any simple $\Gamma$-module $L$ with grade $L=0$ and $\operatorname{r}$.grade $L=n-1$ satisfies that $\mathbb{F}_{n-1} L$ is simple and s.grade $\mathbb{E}_{n-1} L=n$.

Proof. The former assertion was shown in [19, 1.3]. We will show the latter assertion. Put $\mathcal{C}_{\Gamma}:=\{M \in \bmod \Gamma \mid$ s.grade $M \geq n\}$. Then $\mathcal{C}_{\Gamma}\left(\right.$ resp. $\left.\mathcal{C}_{\Gamma^{o p}}\right)$ is an abelian subcategory of $\bmod \Gamma\left(\right.$ resp. $\left.\bmod \Gamma^{o p}\right)$ closed under subfactor modules, and $\mathbb{E}_{n}$ gives a duality between $\mathcal{C}_{\Gamma}$ and $\mathcal{C}_{\Gamma}{ }^{o p}$ such that $\mathbb{E}_{n} \circ \mathbb{E}_{n}$ is isomorphic to the identity functor by [I5, 6.2]. Take a projective resolution $0 \rightarrow P_{n-1} \rightarrow \cdots \rightarrow P_{0} \rightarrow L \rightarrow 0$ by pd $L=n-1$. Taking $\widehat{()}$, we obtain an exact sequence $0 \leftarrow \mathbb{E}_{n-1} L \leftarrow \widehat{P}_{n-1} \leftarrow \cdots \leftarrow$ $\widehat{P}_{0} \leftarrow \widehat{L} \leftarrow 0$ by r.grade $L=n-1$. Thus $\widehat{L} \in \operatorname{ind}\left(\operatorname{pr} \Gamma^{o p}\right)$ holds by gl.dim $\Gamma=n$, and we obtain an exact sequence $0 \rightarrow L \rightarrow \widehat{\widehat{L}} \rightarrow M \rightarrow 0$ with $M:=\mathbb{E}_{n} \mathbb{E}_{n-1} L \in \mathcal{C}_{\Gamma}$ and $\widehat{\widehat{L}} \in \operatorname{ind}(\operatorname{pr} \Gamma)$. Thus $\mathbb{E}_{n-1} L=\mathbb{E}_{n} M \in \mathcal{C}_{\Gamma^{o p}}$ holds, and top $M$ is simple. By the remark above, $\mathbb{F}_{n-1} L=\mathbb{F}_{n} M=\mathbb{E}_{n}(\operatorname{top} M)$ is simple.

3.6.4. Question. When is $\mathbb{F}_{n} L$ a simple $\Gamma^{o p}$-module for a simple $\Gamma$-module $L$ and $n ?$ 


\section{AR QUIVERS}

In representation theory, the concept of additive functions often appears. We recall several results below which assert that some representation theoretic diagrams are characterized by the existence of additive functions:

(a) It is a classical result that Dynkin diagrams and extended Dynkin diagrams are characterized in terms of additive functions $\mathrm{HPR}$.

(b) Brenner characterized AR quivers of representation-finite artin algebras in terms of hammocks, which is a formulation of the existence of additive functions $\mathrm{Br}$. At the same time, Igusa and Todorov gave another characterization independently which does not use additive functions [IT3].

(c) Ringel and Vossieck studied hammocks in [RVo] very clearly, and characterized AR quivers of representation-finite partially ordered sets in terms of hammocks.

(d) Reiten and Van den Bergh characterized AR quivers of representation-finite two-dimensional orders, which essentially uses additive functions [RV].

(e) Inspired by the work of Igusa-Todorov (b), the author characterized AR quivers of representation-finite one-dimensional orders [I5], which does not use additive functions. Then Rump gave another characterization in terms of additive functions R5.

(f) Additive functions are used to characterize rejectable subsets 5.1(2) for twodimensional orders by Reiten and Van den Bergh [RV] and for one-dimensional orders by the author [I2].

In this section, we shall see that (b) and (c) above are understood clearly in our viewpoint of (e) and this paper (see 4.4.1).

4.1. Definition. (1) $Q=\left(Q, Q^{p}, Q^{i}, \tau^{+}, d, d^{\prime}\right)$ is called a translation quiver if $Q$ is a set, $Q^{p}$ and $Q^{i}$ are subsets of $Q, \tau^{+}$is a bijection $Q-Q^{p} \rightarrow Q-Q^{i}, d$ and $d^{\prime}$ are maps $Q \times Q \rightarrow \mathbb{N}_{\geq 0}$ such that $d(Y, X)=d^{\prime}\left(\tau^{+} X, Y\right)$ holds for any $X \in Q-Q^{p}$ and $Y \in Q$, and $d(, X)=0$ implies $X \in Q^{p}$. We call $Q$ admissible if there exists a map $c: Q \rightarrow \mathbb{N}_{>0}$ such that $c(X) d(X, Y)=d^{\prime}(X, Y) c(Y)$ holds for any $X, Y \in Q$. We call $Q$ locally finite if $\sum_{Y \in Q} d(Y, X)<\infty$ and $\sum_{Y \in Q} d^{\prime}(X, Y)<\infty$ hold for any $Y \in Q$.

Usually, we draw $Q$ as a directed graph: $Q$ is the set of vertices, and we draw valued arrows $X \stackrel{\left(d(X, Y), d^{\prime}(X, Y)\right)}{\longrightarrow} Y$ for any $X, Y \in Q$ such that $d(X, Y) \neq 0$, and dotted arrows from $X$ to $\tau^{+} X$ for any $X \in Q-Q^{p}$.

For a $\tau$-category $\mathcal{C}$, we define a locally finite translation quiver $\mathbb{A}(\mathcal{C})=\left(Q, Q^{p}, Q^{i}\right.$, $\left.\tau^{+}, d, d^{\prime}\right)$ called the $A R$ quiver of $\mathcal{C}$ as follows: $Q:=\operatorname{ind} \mathcal{C}, Q^{p}:=\operatorname{ind}_{1}^{+} \mathcal{C}, Q^{i}:=$ $\operatorname{ind}_{1}^{-} \mathcal{C}, d(X, Y)$ is the multiplicity of $X$ in $\theta^{+} Y$ and $d^{\prime}(X, Y)$ is the multiplicity of $Y$ in $\theta^{-} X$. Thus $\mathbb{A}(\mathcal{C})$ displays terms of each $(X]$ and $[X)$ diagrammatically. If $\mathcal{C}$ is a torsionfree class over an artin algebra $\Lambda$ over $R$, then $\mathbb{A}(\mathcal{C})$ is admissible by $k:=R / J_{R}$ and $c(X):=\operatorname{dim}_{k} \operatorname{End}_{\Lambda}(X) / J_{\operatorname{End}_{\Lambda}(X)}$.

(2) For a locally finite translation quiver $Q$, we denote by $\mathbb{Z} Q$ (resp. $\mathbb{N} Q$ ) the free $\mathbb{Z}$-module (resp. free abelian monoid) generated by $Q$. For $X=\sum_{Y \in Q} a_{Y} Y \in \mathbb{Z} Q$, put supp $X:=\left\{Y \in Q \mid a_{Y} \neq 0\right\}$. Define elements $\theta^{+}, \theta^{-}, \tau^{+}$and $\tau^{-}$of $\operatorname{End}_{\mathbb{Z}}(\mathbb{Z} Q)$ as follows: Put $\theta^{+} X:=\sum_{Y \in Q} d(Y, X) Y$ and $\theta^{-} X:=\sum_{Y \in Q} d^{\prime}(X, Y) Y$ for $X \in Q$. Put $\tau^{+} X:=0$ for $X \in Q^{p}, \tau^{-} X:=\left(\tau^{+}\right)^{-1}(X)$ for $X \in Q-Q^{i}$ and $\tau^{-} X:=0$ for $X \in Q^{i}$. When $Q=\mathbb{A}(\mathcal{C})$ for a $\tau$-category $\mathcal{C}$, these definitions are consistent with those in 1.3.1. Define $\phi^{ \pm}$and a (left, right) additive function of $Q$ by a similar 
manner in 1.3.1, and define $\theta_{n}^{+}$and $\eta_{n}^{+} \in \operatorname{End}_{\mathbb{Z}}(\mathbb{Z} Q)(n \geq 0)$ by the recursion formulas in 1.3.3 and 1.4.

4.2. We call an additive category $\mathcal{C}$ with an additive generator $M$ artinian if the ring $\mathcal{C}(M, M)$ is artinian. We call a translation quiver $Q$ artinian (resp. strict) if there exists an artinian (resp. strict) $\tau$-category $\mathcal{C}$ with $Q=\mathbb{A}(\mathcal{C})$. The proposition below gives a simple criterion for $Q$ to be artinian (resp. strict).

Proposition. Let $Q$ be an admissible translation quiver with a finite number of vertices. Then $Q$ is artinian (resp. strict) if and only if any $\tau$-category $\mathcal{C}$ with $Q=\mathbb{A}(\mathcal{C})$ is artinian (resp. strict).

(1) $Q$ is artinian if and only if there exists $n>0$ such that $\theta_{n}^{+}=0$. If any connected component of $Q$ contains a vertex in $Q^{i}$, then $Q$ is artinian if and only if there exists $n>0$ such that $\theta_{n}^{+} X=0$ for any $X \in Q^{i}$.

(2) If $Q$ is artinian, then $Q$ is strict if and only if $Q=\bigcup_{X \in Q^{i}, n \geq 0} \operatorname{supp} \theta_{n}^{+} X$ if and only if $\theta_{n}^{+}=\theta^{+} \circ \theta_{n-1}^{+}-\tau^{+} \circ \theta_{n-2}^{+}$for any $n \geq 2$.

4.2.1. Example. Let $Q$ be the translation quiver below, where $\tau^{+}$is the left translation:

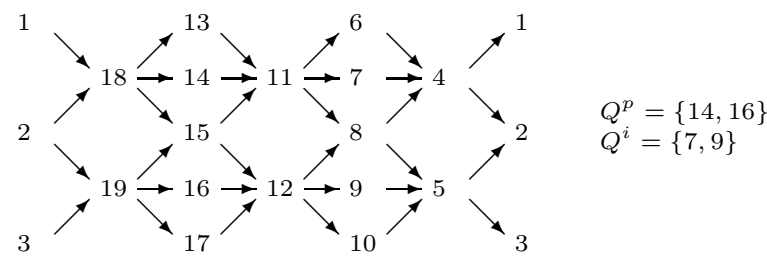

Then the calculation of $\theta_{n}^{+}(7)$ and $\theta_{n}^{+}(9)$ below implies that $Q$ is artinian by 4.2(1), where we describe the diagram in 1.3.3:

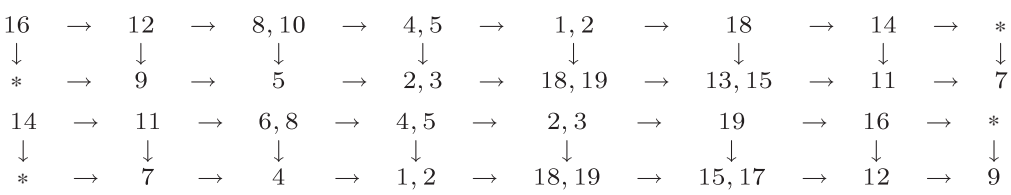

On the other hand, $Q$ is not strict by $4.2(2)$ and $\bigcup_{n \geq 0} \operatorname{supp} \theta_{n}^{+}(7) \cup \operatorname{supp} \theta_{n}^{+}(9)=$ $Q-\{6,8,10,14,16\}$.

4.2.2. Lemma. Let $\mathcal{C}$ be a $\tau$-category with $\#$ ind $\mathcal{C}<\infty$ and $Q=\mathbb{A}(\mathcal{C})$. Assume that any connected component of $Q$ contains a vertex in $Q^{i}$. Then $\mathcal{C}(, X)$ has finite length for any $X \in \mathcal{C}$ if and only if $\mathcal{C}(, X)$ has finite length for any $X \in \operatorname{ind}_{1}^{-} \mathcal{C}$.

Proof. Put $\mathcal{D}:=\{X \in \mathcal{C} \mid \mathcal{C}(, X)$ has finite length $\}, \underline{\mathcal{C}}:=\mathcal{C} /\left[\operatorname{ind}_{1}^{+} \mathcal{C}\right], \overline{\mathcal{C}}:=\mathcal{C} /\left[\operatorname{ind}_{1}^{-} \mathcal{C}\right]$ and $\mathcal{C}^{\prime}:=\operatorname{add}\left(\operatorname{ind}_{1}^{-} \mathcal{C}\right)$. Then $\mathcal{C}^{\prime} \subseteq \mathcal{D}$ holds by our assumption. Fix indecomposable $X \in \mathcal{D}$. Since $\underline{\mathcal{C}}(, X)$ has finite length by $X \in \mathcal{D}, \overline{\mathcal{C}}\left(, \tau^{+} X\right)$ has finite length by the proof of [14, 2.4]. Now we will show $Y:=\tau^{+} X \in \mathcal{D}$. Since we have an exact sequence $0 \rightarrow\left[\mathcal{C}^{\prime}\right](, Y) \rightarrow \mathcal{C}(, Y) \rightarrow \overline{\mathcal{C}}(, Y) \longrightarrow 0$, we only have to show that $\left[\mathcal{C}^{\prime}\right](, Y)$ has finite length. Since any finite length $\mathcal{C}$-module is finitely presented, $\left[\mathcal{C}^{\prime}\right](, Y)$ is a finitely generated $\mathcal{C}$-module. Thus there exists $f \in \mathcal{C}(Z, Y)$ with $Z \in \mathcal{C}^{\prime}$ such that $\mathcal{C}(, Z) \stackrel{\cdot f}{\rightarrow}\left[\mathcal{C}^{\prime}\right](, Y) \rightarrow 0$ is exact. Hence $\left[\mathcal{C}^{\prime}\right](, Y)$ has finite length by $\mathcal{C}^{\prime} \subseteq \mathcal{D}$. Thus $\tau^{+} X \in \mathcal{D}$ holds. Since we have an exact sequence $\mathcal{C}\left(, \tau^{+} X\right) \rightarrow \mathcal{C}\left(, \theta^{+} X\right) \rightarrow \mathcal{C}(, X)$, we obtain $\theta^{+} X \in \mathcal{D}$. Thus any predecessor of $X$ 
in $\mathbb{A}(\mathcal{C})$ is again contained in $\mathcal{D}$. By our assumption, we can easily show that there exists a path from any vertex in $Q$ to some vertex in $Q^{i}$. Thus $\mathcal{D}=\mathcal{C}$ holds.

4.2.3. Proof of 4.2. (1) (cf. [13, 7.3]) The former assertion follows from 1.3.3, and the latter assertion follows from the former one and 4.2.2. (2) follows from [13, 7.4].

Now the first equivalence follows from (1) and (2), where we remark that any admissible translation quiver is realized as the AR quiver of some $\tau$-category [I5] 4.2.1].

4.3. Theorem. Let $\Gamma$ be an artin algebra and $\mathcal{C}:=\operatorname{pr} \Gamma$. Put

$$
l_{X}(Y):=\operatorname{length}_{\mathcal{C}(X, X)} \mathcal{C}(X, Y)
$$

for any $X, Y \in \mathcal{C}$.

(1) Assume that the conditions in 2.1 are satisfied. Then $\phi^{+}(\mathbb{N}$ ind $\mathcal{C}) \supseteq \mathbb{N}$ ind $\mathcal{C}$ holds. Moreover, a map $l$ : ind $\mathcal{C} \rightarrow \mathbb{N}_{>0}$ is a right additive function if and only if $l=\sum_{X \in \operatorname{ind}_{1}^{+} \mathcal{C}} a_{X} l_{X}$ holds for some $\left(a_{X}\right) \in \mathbb{N}^{\operatorname{ind}_{1}^{+} \mathcal{C}}$. Such $\left(a_{X}\right)$ is uniquely determined.

(2) Assume that the conditions in 3.1 are satisfied and put $\mathfrak{S}^{+}(\mathcal{C}):=\{X \in$ $\operatorname{ind}_{1}^{+} \mathcal{C} \mid \mu_{X}^{+}$is not an epimorphism $\}$. Then a map $l: \operatorname{ind} \mathcal{C} \rightarrow \mathbb{N}_{>0}$ is an additive function if and only if $l=\sum_{X \in \mathfrak{S}^{+}(\mathcal{C})} a_{X} l_{X}$ holds for some $\left(a_{X}\right) \in \mathbb{N}^{\mathfrak{S}^{+}(\mathcal{C})}$. Such $\left(a_{X}\right)$ is uniquely determined.

Proof. Since $0 \rightarrow \mathcal{C}\left(X, \tau^{+} Y\right) \rightarrow \mathcal{C}\left(X, \theta^{+} Y\right) \rightarrow \mathcal{J}_{\mathcal{C}}(X, Y) \rightarrow 0$ is exact for any $Y \in \mathcal{C}$, we obtain $l_{X}\left(\phi^{+} Y\right)=0$ for any $Y \in$ ind $\mathcal{C}-\{X\}$ and $l_{X}\left(\phi^{+} X\right)=1$.

(1) Since $\mathcal{C}$ is artinian strict,

$$
\begin{aligned}
\phi^{+}\left(\sum_{n \geq 0} \theta_{n}^{+} X\right) & =\sum_{n \geq 0} \theta_{n}^{+} X-\sum_{n \geq 0}\left(\theta^{+} \theta_{n}^{+} X-\tau^{+} \theta_{n-1}^{+} X\right) \\
& =\sum_{n \geq 0} \theta_{n}^{+} X-\sum_{n \geq 0} \theta_{n+1}^{+} X=X
\end{aligned}
$$

holds for any $X \in \mathcal{C}$ by $4.2(2)$. Thus the first assertion follows. We will show the "only if" part. Put $a_{X}:=l\left(\phi^{+} X\right) \geq 0$ for any $X \in \operatorname{ind}_{1}^{+} \mathcal{C}$ and $l^{\prime}:=l-$ $\sum_{X \in \operatorname{ind}_{1}^{+} \mathcal{c}} a_{X} l_{X}$. Since $l^{\prime} \circ \phi^{+}=0$ holds, we obtain $l^{\prime}=0$ by the first assertion. Thus $l=\sum_{X \in \operatorname{ind}_{1}^{+} \mathcal{C}} a_{X} l_{X}$ holds. The third assertion follows from the first.

(2) Since $l\left(\phi^{+} X\right)=0$ holds for any $X \in \operatorname{ind}_{1}^{+} \mathcal{C}-\mathfrak{S}^{+}(\mathcal{C})$ by 3.2(3), we obtain $l=$ $\sum_{X \in \mathfrak{S}^{+}(\mathcal{C})} a_{X} l_{X}$ by $(1)$. Fix $X \in \mathfrak{S}^{+}(\mathcal{C})$. We only have to show that $l_{X}\left(\mu_{Y}^{-}\right) \geq 0$ holds for any $Y \in \operatorname{ind}_{1}^{-} \mathcal{C}$. Put $Z:=\mathrm{n}^{-}(Y) \in$ ind $\mathcal{C}$; then $l_{X}\left(\mu_{Y}^{-}\right)=l_{X}\left(\mu_{Z}^{+}\right)$holds by 1.4. Since $\mathrm{n}^{+}(X) \notin \operatorname{ind}_{1}^{-} \mathcal{C}$ holds by $3.2(3)$, we obtain $X \neq Z$. Thus $l_{X}\left(\phi^{+} Z\right)=0$ implies $l_{X}\left(\mu_{Z}^{+}\right)=l_{X}\left(\tau^{+} Z\right) \geq 0$.

4.4. Theorem. Let $Q$ be an admissible artinian translation quiver with a finite number of vertices. Then the following conditions (1-i), (2-i), (3-i) and (4-i) are equivalent for each $i(1 \leq i \leq 4)$.

(1) There is an artin algebra $\Lambda$

(1-1) with a torsion theory $(\mathcal{T}, \mathcal{F})$ on $\bmod \Lambda$ such that $Q=\mathbb{A}(\mathcal{F})$,

(1-2) with a hereditary torsion theory $(\mathcal{T}, \mathcal{F})$ on $\bmod \Lambda$ such that $Q=\mathbb{A}(\mathcal{F})$,

(1-3) such that $Q=\mathbb{A}(\bmod \Lambda)$,

(1-4) such that $Q=\mathbb{A}\left(\bmod _{s p} \Lambda\right)$. 
(2) Any $\tau$-category $\mathcal{C}$ with $\mathbb{A}(\mathcal{C})=Q$ is

(2-1) strict,

(2-2) strict and $\mathrm{n}^{-}$gives a map $\operatorname{ind}_{1}^{-} \mathcal{C}-\operatorname{ind}_{0}^{-} \mathcal{C} \rightarrow$ ind $\mathcal{C}$,

(2-3) strict and $\mathrm{n}^{-}$gives a map $\operatorname{ind}_{1}^{-} \mathcal{C}-\operatorname{ind}_{0}^{-} \mathcal{C} \rightarrow \operatorname{ind} \mathcal{C}-\operatorname{ind}_{1}^{+} \mathcal{C}$,

(2-4) strict and $\mathrm{n}^{-}$gives a map $\operatorname{ind}_{1}^{-} \mathcal{C}-\operatorname{ind}_{0}^{-} \mathcal{C} \rightarrow \operatorname{ind}_{1}^{+} \mathcal{C}-\operatorname{ind}_{0}^{+} \mathcal{C}$.

(3) Q satisfies

(3-1) $Q=\bigcup_{X \in Q^{i}, n \geq 0} \operatorname{supp} \theta_{n}^{+} X$,

(3-2) $Q=\bigcup_{X \in Q^{i}, n \geq 0} \operatorname{supp} \theta_{n}^{+} X$, and for any $X \in Q^{i}$ with $\theta^{-} X \neq 0$, there exists $n \geq 0$ such that $\eta_{i}^{+} X \in \mathbb{N}\left(Q-Q^{p}\right)$ for any $i(0 \leq i<n)$ and $\eta_{n+1}^{+} X=0$

(3-3) (3-2) and $\eta_{n}^{+} X \in Q-Q^{p}$,

(3-4) (3-2) and $\eta_{n}^{+} X \in Q^{p}$.

(4) $Q$ has

(4-1) a right additive function $l$,

(4-2) an additive function $l$,

(4-3) an additive function $l$ with $l^{-}=\operatorname{ind}_{1}^{-} \mathcal{C}$,

(4-4) an additive function $l$ with $l^{-}=\operatorname{ind}_{0}^{-} \mathcal{C}$.

4.4.1. Remark. (1) In 4.4(2), we can replace "any" by "some". In 4.4(3-2), n- $(X)=$ $\eta_{n}^{+} X$ holds. In 4.4(1), we can add the condition that $\Lambda$ is a finite-dimensional algebra over an arbitrary finite field $k$ (see [15 4.2.1]).

(2) Our condition (3-3) simplifies that of Igusa-Todorov (b) above, and our condition (4-3) simplifies that of Brenner (b) above. After the work of Brenner, Ringel and Vossieck (c) call a simply connected translation quiver $Q$ with a unique source $X$ hammock if $Q$ has an additive function $l$ with $l^{-}=\{X\}$. Thus our condition (4-4) is a generalization of their hammock condition to a general translation quiver.

4.4.2. Proof of 4.4. We can fix an artin algebra $\Gamma_{0}$ such that $\mathcal{C}_{0}:=\operatorname{pr} \Gamma_{0}$ forms a $\tau$-category with $Q=\mathbb{A}\left(\mathcal{C}_{0}\right)$ by $[$ I5 4.2 .1$]$. Then $(1-i) \Rightarrow(4-i) \Rightarrow(2-i) \Rightarrow\left(\mathcal{C}:=\mathcal{C}_{0}\right.$ satisfies $(2-i)) \Rightarrow(1-i)$ holds by $2.1(i=1), 3.1(i=2), 3.3(i=3)$ and $3.4(i=4)$. Moreover, $(2-i) \Leftrightarrow(3-i)$ holds by $4.2(1)(i=1)$ and 1.4.2(1) $(i=2,3,4)$.

4.5. Example. (1) Let $Q$ be the artinian strict translation quiver below, where $\tau^{+}$ is the left translation:

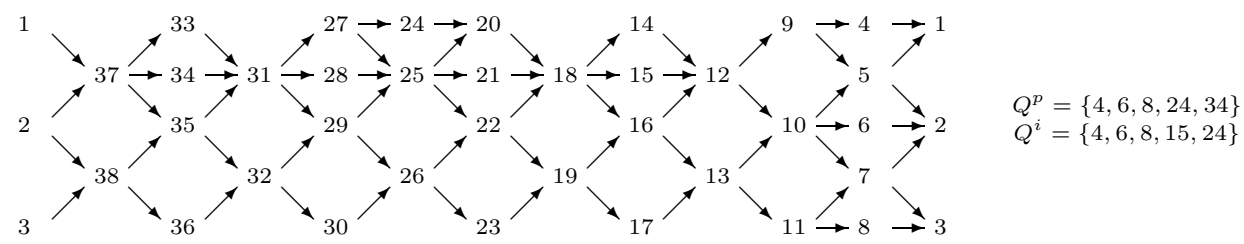

Then the calculation of $\eta_{n}^{+}\left(Q^{i}\right)$ below shows that $Q$ satisfies condition 4.4(3-2), where we describe the diagram in 1.4. Thus $Q=\mathbb{A}(\mathcal{C})$ holds for some hereditary 
torsionfree class $\mathcal{C}$ over an artin algebra $\Lambda$.

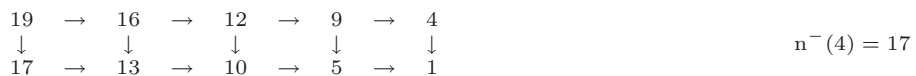

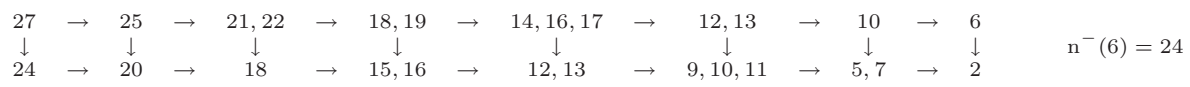

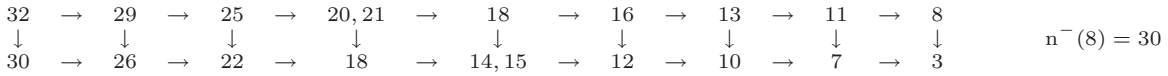

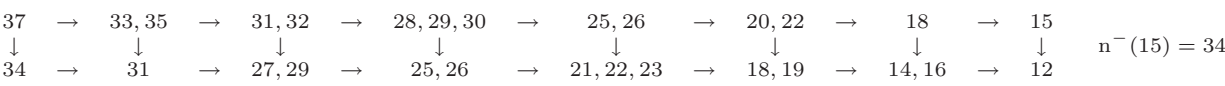

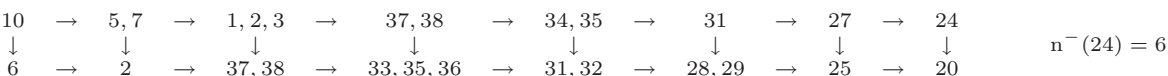

(2) Let $Q$ be the artinian strict translation quiver below, where $\tau^{+}$is the left translation:

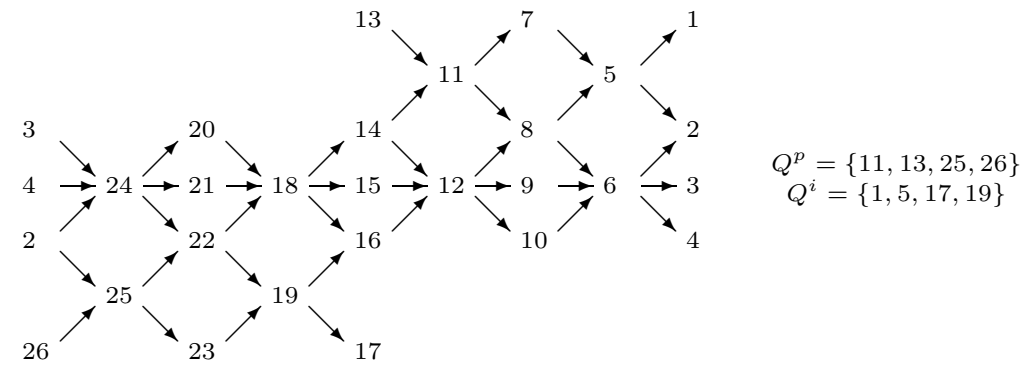

Then the calculation of $\eta_{n}^{+}\left(Q^{i}\right)$ below shows that $Q$ satisfies condition $4.4(3-4)$, where we describe the diagram in 1.4. Thus $Q=\mathbb{A}\left(\bmod _{s p} \Lambda\right)$ holds for some artin algebra $\Lambda$.

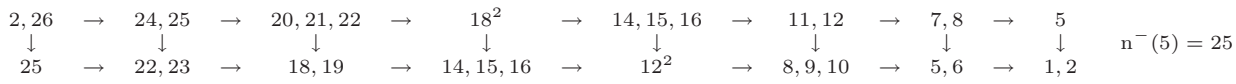

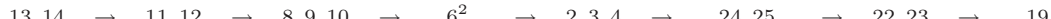

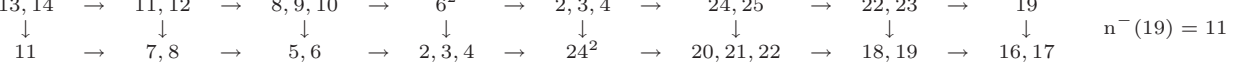

(3) Let $Q$ be the artinian strict translation quiver below, where $\tau^{+}$is the left translation:

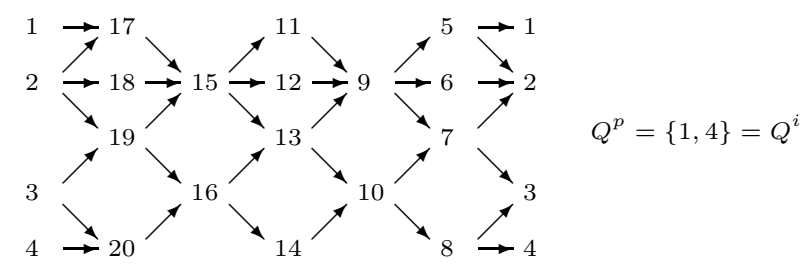

Then the calculation of $\eta_{n}^{+}\left(Q^{i}\right)$ below shows that $Q$ satisfies condition $4.4(3-3)$, where we describe the diagram in 1.4.

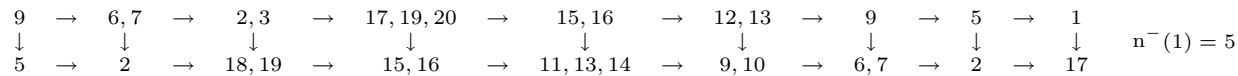

$$
\begin{aligned}
& \begin{array}{cccccccccccccccccc}
10 & \rightarrow & 7 & \rightarrow & 2 & \rightarrow & 17,18 & \rightarrow & 15 & \rightarrow & 13 & \rightarrow & 10 & \rightarrow & 8 & \rightarrow & 4 \\
\downarrow & & \downarrow & & \downarrow & & \downarrow & & \downarrow & & \downarrow & & \downarrow & & \downarrow & & \downarrow & \\
8 & \rightarrow & 3 & \rightarrow & 19 & \rightarrow & 15 & \rightarrow & 11,12 & \rightarrow & 9 & \rightarrow & 7 & \rightarrow & 3 & \rightarrow & 20 &
\end{array} \quad \mathrm{n}^{-}(4)=8
\end{aligned}
$$

Thus $Q=\mathbb{A}(\bmod \Lambda)$ holds for some artin algebra $\Lambda$. 
(4) Let $Q$ be the artinian strict translation quiver below, where $\tau^{+}$is the left translation. Then $Q$ is a part of the preprojective component of the hereditary algebra $\Lambda$ of the wild quiver $\stackrel{i}{\bullet} \dot{\uparrow}^{\bullet}, \bullet \leftarrow \rightarrow \bullet$, and $Q=\mathbb{A}(\mathcal{C})$ holds for a faithful torsionfree class $\mathcal{C}$ of $\bmod \Lambda$.

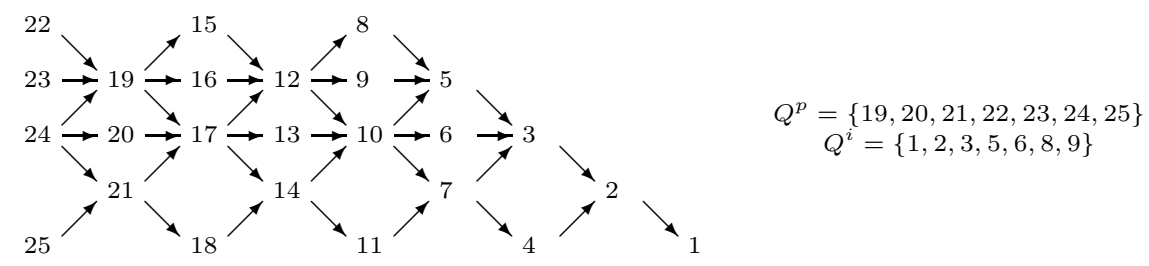

In fact, $Q$ satisfies the condition 4.4(3-1), but not (3-2) since the calculation below shows that $\mathrm{n}^{-}(i)$ is not defined for $i=5,6,8,9$.

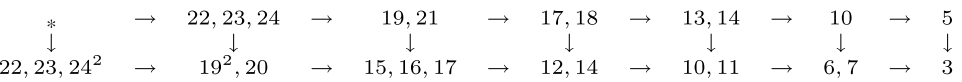

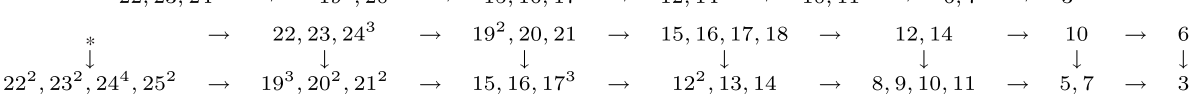

$$
\begin{aligned}
& * \quad \rightarrow \quad 22,24^{2}, 25 \rightarrow 19,20,21 \rightarrow 16,17 \rightarrow 12 \rightarrow 8
\end{aligned}
$$

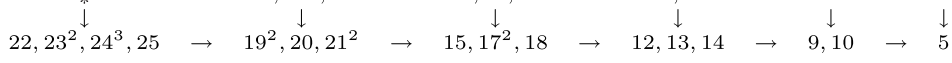

$$
\begin{aligned}
& * \quad \rightarrow \quad 23,24^{2}, 25 \quad \rightarrow \quad 19,20,21 \quad \rightarrow \quad 15,17 \quad \rightarrow \quad 12 \quad \rightarrow \quad 9
\end{aligned}
$$

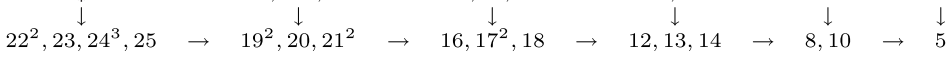

\section{REJECTION THEORY}

The rejection theory of an additive category $\mathcal{C}$ is a study of subcategories called rejective (5.1) and the corresponding subsets of ind $\mathcal{C}$ called rejectable (5.1). For example, rejective subcategories of $\bmod \Lambda$ for an artin algebra $\Lambda$ are given by factor algebras of $\Lambda$ (5.1.1). The first example of rejection theory seems to be the DK (=Drozd-Kirichenko) Rejection Lemma [DK] (see 5.3.1), which characterizes one-point rejectable subsets and plays a crucial role in the theory of Bass orders [DKR, [Ro, [HN]. In [I1], the author studied the rejection theory of orders and artin algebras by connecting with Auslander-Reiten theory, and characterized finite rejectable subsets in terms of AR quivers (see 5.3), a generalization of the DK Rejection Lemma. It is surprising that the rejectability of a finite subset $\mathbf{S}$ of ind $\mathcal{C}$ depends only on the restriction of $\mathbb{A}(\mathcal{C})$ to $\mathbf{S}$ (see [1] for examples of rejectable subsets). Moreover, he studied the rejection theory of arbitrary $\tau$-categories in [14]. In particular, he generalized results in [11] to $\tau$-categories, and successfully applied to characterize AR quivers of representation-finite orders [15]. Of course, his results are valid for our case when $\mathcal{C}$ is a torsionfree class of $\bmod \Lambda$, and we will give a representation theoretic interpretation of rejective subcategories of such $\mathcal{C}$ in 5.2. Note that, recently, rejective subcategories were successfully applied to quite different kinds of problems, Solomon's second conjecture on zeta functions of orders [17] and finiteness of representation dimension of artin algebras [18].

5.1. Definition. In the rest of this paper, assume that any subcategory is full and closed under isomorphism, direct sums and direct summands. Let $\mathcal{C}^{\prime}$ be a subcategory of a Krull-Schmidt category $\mathcal{C}$. 
(1) [14 5.1] We call $\mathcal{C}^{\prime}$ a rejective subcategory of $\mathcal{C}$ if the inclusion functor $\mathcal{C}^{\prime} \rightarrow \mathcal{C}$ has a right adjoint ()$^{-}: \mathcal{C} \rightarrow \mathcal{C}^{\prime}$ with a counit $\epsilon^{-}$such that $\epsilon_{X}^{-}$is a monomorphism for any $X \in \mathcal{C}$, and a left adjoint ()$^{+}: \mathcal{C} \rightarrow \mathcal{C}^{\prime}$ with a unit $\epsilon^{+}$such that $\epsilon_{X}^{+}$is an epimorphism for any $X \in \mathcal{C}$ (compare with torsion theories 1.2). We call $\mathcal{C}^{\prime}$ a trivial subcategory of $\mathcal{C}$ if $\mathcal{C}$ is a unique rejective subcategory of $\mathcal{C}$ which contains $\mathcal{C}^{\prime}$.

(2) [14, 8.2] We call a subset $\mathbf{S}$ of ind $\mathcal{C}$ rejectable (resp. trivial) if $\mathbf{S}=\operatorname{ind} \mathcal{C}$-ind $\mathcal{C}^{\prime}$ for some rejective (resp. trivial) subcategory $\mathcal{C}^{\prime}$ of $\mathcal{C}$.

5.1.1. Example ([14, 5.4]). Let $\Lambda$ be an artin algebra and $\mathcal{C}=\bmod \Lambda$.

Any ring morphism $G: \Lambda \rightarrow \Gamma$ induces a faithful functor $G^{*}: \bmod \Gamma \rightarrow \mathcal{C}$, which is full if $G$ is surjective. A subcategory $\mathcal{C}^{\prime}$ of $\mathcal{C}$ is rejective if and only if there exists a factor algebra $\Gamma$ of $\Lambda$ such that $\mathcal{C}^{\prime}=\bmod \Gamma$. In this case, adjoint functors are given by ()$^{+}=\Gamma \otimes_{\Lambda}$ and ()$^{-}=\operatorname{Hom}_{\Lambda}(\Gamma$,$) . Thus we obtain a bijection from$ factor algebras of $\Lambda$ to rejective subcategories of $\mathcal{C}$ defined by $\Gamma \mapsto \bmod \Gamma$.

Note that it is shown in [14,6.3] that any rejective subcategory $\mathcal{C}^{\prime}$ of a (strict) $\tau$-category $\mathcal{C}$ forms a (strict) $\tau$-category again if $\mathcal{C} /\left[\mathcal{C}^{\prime}\right]$ is artinian.

5.2. Theorem. Let $\Lambda$ be an artin algebra, $(\mathcal{T}, \mathcal{C})$ a faithful torsion theory on $\bmod \Lambda$ and $\mathcal{C}^{\prime}$ a subcategory of $\mathcal{C}$. Then $\mathcal{C}^{\prime}$ is a rejective subcategory of $\mathcal{C}$ if and only if there exists a morphism $G: \Lambda \rightarrow \Gamma$ of artin algebras such that $\mathcal{C}^{\prime}=\mathcal{C} \cap \operatorname{Fac} \Gamma$, $\Gamma \in \mathcal{C}^{\prime}$ and $\Gamma / G(\Lambda) \in \mathcal{T}$, where we denote by $\operatorname{Fac} \Gamma$ the full subcategory of $\bmod \Lambda$ consisting of factor modules of $\Gamma^{n}(n>0)$. In this case, $G^{*}$ induces an equivalence from a faithful torsionfree class $\left\{X \in \bmod \Gamma \mid G^{*} X \in \mathcal{C}\right\}$ on $\bmod \Gamma$ to $\mathcal{C}^{\prime}$.

Proof. (i) We will show the "only if" part.

Let ()$^{+}: \mathcal{C} \rightarrow \mathcal{C}^{\prime}$ be a left adjoint of the inclusion functor with a unit $\epsilon^{+}$, and let $\Gamma:=\operatorname{End}_{\Lambda}\left(\Lambda^{+}\right)$. Then $\epsilon_{\Lambda}^{+} \in \operatorname{Hom}_{\Lambda}\left(\Lambda, \Lambda^{+}\right)$is given by a left multiplication of an element $a \in \Lambda^{+}$. Taking $\operatorname{Hom}_{\Lambda}\left(, \Lambda^{+}\right)$, we obtain a bijection $(a \cdot): \operatorname{End}_{\Lambda}\left(\Lambda^{+}\right)=\Gamma \rightarrow$ $\operatorname{Hom}_{\Lambda}\left(\Lambda, \Lambda^{+}\right)=\Lambda^{+}$. Thus a map $G: \Lambda \rightarrow \Gamma$ is well defined by $x a=a G(x)$ for any $x \in \Lambda$. Obviously $G$ is a ring morphism. Since $(a \cdot)$ is a bijection such that $(a \cdot) \circ G=$ $(\cdot a)=\epsilon_{\Lambda}^{+}$, we can replace $\Lambda^{+}$and $\epsilon_{\Lambda}^{+}$by $\Gamma$ and $G$. Let $\Lambda \stackrel{G}{\rightarrow} \Gamma \stackrel{H}{\rightarrow} \Gamma / G(\Lambda) \rightarrow 0$ be exact. Taking $\operatorname{Hom}_{\Lambda}(, X)$ for any $X \in \mathcal{C}$, we obtain $\Gamma / G(\Lambda) \in{ }^{\perp} \mathcal{C}=\mathcal{T}$. For any $X \in \mathcal{C} \cap \operatorname{Fac} \Gamma$, take a surjection $f \in \operatorname{Hom}_{\Lambda}\left(\Gamma^{n}, X\right)$. Since $f$ factors through the injection $\epsilon_{X}^{-} \in \operatorname{Hom}_{\Lambda}\left(X^{-}, X\right)$, we obtain $X=X^{-} \in \mathcal{C}^{\prime}$. Conversely, for any $X \in \mathcal{C}^{\prime}$, take a surjection $f \in \operatorname{Hom}_{\Lambda}\left(\Lambda^{n}, X\right)$. Since $f^{+} \in \operatorname{Hom}_{\Lambda}\left(\Gamma^{n}, X\right)$ is surjective again, we obtain $X \in \mathcal{C} \cap \operatorname{Fac} \Gamma$. Thus $\mathcal{C}^{\prime}=\mathcal{C} \cap \operatorname{Fac} \Gamma$ holds.

(ii) We will show the "if" part.

Fix any $X \in \mathcal{C}$. Put $X^{-}:=\operatorname{Hom}_{\Lambda}(\Gamma, X)$. Then the natural map $\epsilon_{X}^{-} \in$ $\operatorname{Hom}_{\Lambda}\left(X^{-}, X\right)$ is injective by $\Gamma / G(\Lambda) \in \mathcal{T}$. Thus $X^{-}$is a unique maximal submodule of $X$ such that $X^{-} \in \operatorname{Fac} \Gamma$. Hence $X^{-} \in \mathcal{C} \cap \operatorname{Fac} \Gamma=\mathcal{C}^{\prime}$ holds, and $\operatorname{Hom}_{\Lambda}\left(, X^{-}\right) \stackrel{{ }^{-} \epsilon_{X}^{-}}{\longrightarrow} \operatorname{Hom}_{\Lambda}(, X)$ is an isomorphism on $\operatorname{Fac} \Gamma \supseteq \mathcal{C}^{\prime}$. Thus ()$^{-}: \mathcal{C} \rightarrow \mathcal{C}^{\prime}$ gives a right adjoint of the inclusion functor with a counit $\epsilon^{-}$. Let $\mathbb{F}: \bmod \Lambda \rightarrow \mathcal{C}$ be the left adjoint of the inclusion functor $(1.2), X^{+}:=\mathbb{F}\left(\Gamma \otimes_{\Lambda} X\right) \in \mathcal{C} \cap \mathrm{Fac} \Gamma=\mathcal{C}^{\prime}$ and $\epsilon_{X}^{+} \in \operatorname{Hom}_{\Lambda}\left(X, X^{+}\right)$the natural map. Then $\operatorname{Hom}_{\Lambda}\left(X^{+}, Y\right)=\operatorname{Hom}_{\Lambda}\left(\Gamma \otimes_{\Lambda} X, Y\right)=$ $\operatorname{Hom}_{\Lambda}\left(X, Y^{-}\right)=\operatorname{Hom}_{\Lambda}(X, Y)$ holds for any $Y \in \mathcal{C}^{\prime}$. Thus ()$^{+}: \mathcal{C} \rightarrow \mathcal{C}^{\prime}$ gives a left adjoint of the inclusion functor with a unit $\epsilon^{+}$.

(iii) We will show the latter assertion.

Obviously $\mathcal{F}^{\prime}:=\left\{X \in \bmod \Gamma \mid G^{*} X \in \mathcal{C}\right\}$ forms a torsionfree class on $\bmod \Gamma$ and $G^{*}$ induces a functor $\mathcal{F}^{\prime} \rightarrow \mathcal{C} \cap F a c \Gamma=\mathcal{C}^{\prime}$. For any $X \in \mathcal{C}^{\prime}$, we have an isomorphism 
$\operatorname{Hom}_{\Lambda}(\Gamma, X)=X^{-} \stackrel{\epsilon_{X}^{-}}{\rightarrow} X$ by (ii). Since $\Gamma$ is a $(\Lambda, \Lambda)$-bimodule, we can regard $X^{-}=\operatorname{Hom}_{\Lambda}(\Gamma, X)$ as a $\Gamma$-module such that $G^{*}\left(X^{-}\right)=X$. Thus $G^{*}: \mathcal{F}^{\prime} \rightarrow \mathcal{C}^{\prime}$ is dense. Finally we will show that $G^{*}$ is full faithful on $\mathcal{F}^{\prime}$. For any $X, Y \in \mathcal{F}^{\prime}$, take an exact sequence $\Gamma^{m} \rightarrow \Gamma^{n} \rightarrow X \rightarrow 0$ in $\bmod \Gamma$. Taking $\operatorname{Hom}_{\Lambda}(, Y)$ and $\operatorname{Hom}_{\Gamma}(, Y)$, we obtain $\operatorname{Hom}_{\Lambda}(X, Y)=\operatorname{Hom}_{\Gamma}(X, Y)$ by $Y=\operatorname{Hom}_{\Lambda}(\Gamma, Y)$.

5.3. Let $\mathcal{C}$ be a $\tau$-category, $\mathcal{C}^{\prime}$ a subcategory of $\mathcal{C}$, and $\overline{\mathcal{C}}:=\mathcal{C} /\left[\mathcal{C}^{\prime}\right]$ the factor category. Then we can regard ind $\mathcal{C}$ as a disjoint union of ind $\overline{\mathcal{C}}$ and ind $\mathcal{C}^{\prime}$ naturally. By [14 1.4], $\overline{\mathcal{C}}$ forms a $\tau$-category again, and $\mathbb{A}(\overline{\mathcal{C}})$ is obtained by deleting vertices in ind $\mathcal{C}^{\prime}$ from $\mathbb{A}(\mathcal{C})$. Thus we easily obtain the terms $\theta_{\overline{\mathcal{C}}}^{ \pm}$and $\tau_{\overline{\mathcal{C}}}^{ \pm}$of left and right $\tau$-sequences in $\overline{\mathcal{C}}$. Notice that the non-trivial subcategory $\mathcal{C}^{\prime}$ of $\mathcal{C}$ is rejective if any subcategory of $\mathcal{C}$ containing $\mathcal{C}^{\prime}$ is trivial except $\mathcal{C}^{\prime}$. Thus we can use both (1) and (2) below to check the rejectivity.

Theorem. Let $\mathcal{C}^{\prime}$ be a rejective subcategory of a strict $\tau$-category $\mathcal{C}$. Assume that $\overline{\mathcal{C}}:=\mathcal{C} /\left[\mathcal{C}^{\prime}\right]$ is artinian.

(1) [I4, 8.2.1] $\mathcal{C}^{\prime}$ is a trivial subcategory of $\mathcal{C}$ if and only if $\overline{\mathcal{C}}(B, A)=0$ holds for any $A \in \operatorname{ind}_{1}^{-} \mathcal{C}$ and $B \in \operatorname{ind}_{1}^{+} \mathcal{C}$ if and only if the condition below is satisfied:

For any $X \in \operatorname{ind} \overline{\mathcal{C}} \cap \operatorname{ind}_{1}^{-} \mathcal{C}$, put

$$
Y_{0}:=X, \quad Y_{1}:=\theta_{\overline{\mathcal{C}}}^{+} X \quad \text { and } \quad Y_{i}:=\left(\theta_{\overline{\mathcal{C}}}^{+} Y_{i-1}-\tau_{\overline{\mathcal{C}}}^{+} Y_{i-2}\right)_{+}
$$

for $i \geq 2$. Then $Y_{i} \in \mathbb{Z}\left(\operatorname{ind} \overline{\mathcal{C}}-\operatorname{ind}_{1}^{+} \mathcal{C}\right)$ holds for any $i \geq 0$.

(2) [I4, 8.2.2] $\mathcal{C}^{\prime}$ is a rejective subcategory of $\mathcal{C}$ if and only if $\mu_{A}^{-}$is a monomorphism and $\mu_{B}^{+}$is an epimorphism in $\overline{\mathcal{C}}$ for any $A \in \operatorname{ind} \overline{\mathcal{C}}-\operatorname{ind}_{1}^{-} \mathcal{C}$ and $B \in$ ind $\overline{\mathcal{C}}-\operatorname{ind}_{1}^{+} \mathcal{C}$ if and only if (i) and (ii) below are satisfied:

(i) For any $X \in \operatorname{ind} \overline{\mathcal{C}}-\operatorname{ind}_{1}^{-} \mathcal{C}$, put $Y_{0}:=\theta_{\overline{\mathcal{C}}}^{-} X, Y_{1}:=\theta_{\overline{\mathcal{C}}}^{+} \theta_{\overline{\mathcal{C}}}^{-} X-X$ and $Y_{i}:=\theta_{\overline{\mathcal{C}}}^{+} Y_{i-1}-\tau_{\overline{\mathcal{C}}}^{+} Y_{i-2}$ for $i \geq 2$. Then $Y_{i} \in \mathbb{N}$ ind $\overline{\mathcal{C}}$ holds for any $i \geq 0$.

(ii) For any $X \in \operatorname{ind} \overline{\mathcal{C}}-\operatorname{ind}_{1}^{+} \mathcal{C}$, put $Y_{0}:=\theta_{\overline{\mathcal{C}}}^{+} X, Y_{1}:=\theta_{\overline{\mathcal{C}}}^{-} \theta_{\overline{\mathcal{C}}}^{+} X-X$ and $Y_{i}:=\theta_{\overline{\mathcal{C}}}^{-} Y_{i-1}-\tau_{\overline{\mathcal{C}}}^{-} Y_{i-2}$ for $i \geq 2$. Then $Y_{i} \in \mathbb{N}$ ind $\overline{\mathcal{C}}$ holds for any $i \geq 0$.

5.3.1. Corollary (DK Rejection Lemma). Let $\mathcal{C}^{\prime}$ be a rejective subcategory of a strict $\tau$-category $\mathcal{C}$. Assume that ind $\mathcal{C}-$ ind $\mathcal{C}^{\prime}=\{X\}$ and $\mathcal{C} /\left[\mathcal{C}^{\prime}\right]$ is artinian. Then $\mathcal{C}^{\prime}$ is a rejective subcategory of $\mathcal{C}$ if and only if $X \in \operatorname{ind}_{1}^{+} \mathcal{C} \cap \operatorname{ind}_{1}^{-} \mathcal{C}$ holds.

5.4. Example. (1) By 5.3.1, singleton sets $\{4\},\{6\},\{8\},\{24\}$ in 4.5(1) and $\{1\}$, $\{4\}$ in $4.5(3)$ are rejectable.

(2) We can easily check that $\begin{array}{rlll}11 \rightarrow 8 \rightarrow 6 & \rightarrow & 3 \\ \downarrow & 1 & 1 \\ 4 & \rightarrow & 24 \rightarrow 22 \rightarrow 19\end{array}$ in 4.5(2) is rejectable by $5.3(2)$ (or (1)). Moreover, the $\mathrm{AR}$ quiver $\mathbb{A}\left(\mathcal{C}^{\prime}\right)$ of the corresponding rejective subcategory $\mathcal{C}^{\prime}$ is the following:

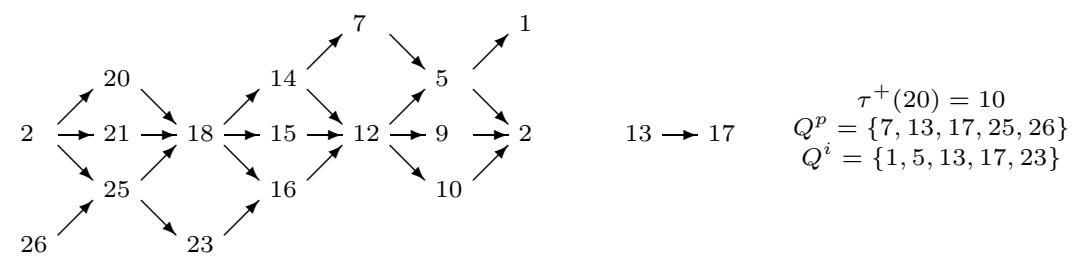




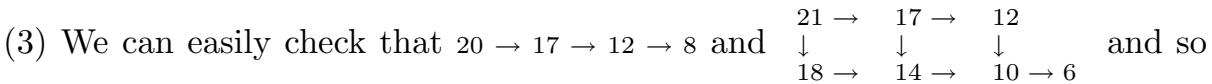
on in $4.5(4)$ are rejectable.

5.5. Let $\mathcal{C}^{\prime}$ be a rejective subcategory of a strict $\tau$-category $\mathcal{C}$. If $\overline{\mathcal{C}}:=\mathcal{C} /\left[\mathcal{C}^{\prime}\right]$ is artinian, then $\overline{\mathcal{C}}$ forms a strict $\tau$-category again by [I4, 6.1] (cf. 5.3(2)). In particular, we obtain the following result by 2.1 .

Proposition. Let $\mathcal{C}$ be a faithful torsionfree class over an artin algebra $\Lambda, \mathcal{C}^{\prime}$ a rejective subcategory of $\mathcal{C}$ and $\overline{\mathcal{C}}:=\mathcal{C} /\left[\mathcal{C}^{\prime}\right]$. If $\#$ ind $\overline{\mathcal{C}}<\infty$, then $\overline{\mathcal{C}}$ is equivalent to a faithful torsionfree class over some artin algebra $\Lambda^{\prime}$.

5.5.1. The above result 5.5 holds even if we drop the assumption $\#$ ind $\overline{\mathcal{C}}<\infty$. See the author's forthcoming papers.

\section{REFERENCES}

[A] M. Auslander: Representation theory of Artin algebras. I, II. Comm. Algebra 1 (1974), 177-268; ibid. 1 (1974), 269-310. MR 50:2240

[AB] M. Auslander, M. Bridger: Stable module theory. Memoirs of the A merican Mathematical Society, No. 94 American Mathematical Society, Providence, R.I. 1969146 pp. MR 42:4580

[AR1] M. Auslander, I. Reiten: Representation theory of Artin algebr as. III. Almost split sequences. Comm. Algebra 3 (1975), 239-294. MR 52:504

[AR2] M. Auslander, I. Reiten: $k$-Gorenstein algebras and syzygy modules. J. Pure Appl. Algebra 92 (1994), no. 1, 1-27. MR 95d:16008

[AR3] M. Auslander, I. Reiten: Syzygy modules for Noetherian rings. J. Algebra 183 (1996), no. 1, 167-185. MR 97g:16012

[ARS] M. Auslander, I. Reiten, S. O. Smalø: Representation theory of Artin algebras. Cambridge Studies in Advanced Mathematics, 36. Cambridge University Press, Cambridge, 1995. MR 96c:16015

[AS] M. Auslander, S. O. Smalø: Almost split sequences in subcategories. J. Algebra 69 (1981), no. 2, 426-454. MR 82j:16048a

[As] I. Assem: Tilting theory-an introduction. Topics in algebra, Part 1 (Warsaw, 1988), 127-180, Banach Center Publ., 26, Part 1, PWN, Warsaw, 1990. MR 93g:16011

[B] H. Bass: On the ubiquity of Gorenstein rings. Math. Z. 821963 8-28. MR 27:3669

[Bj] J-E. Bjork: The Auslander condition on Noetherian rings. Seminaire d'Algebre Paul Dubreil et Marie-Paul Malliavin, 39eme Annee (Paris, 1987/1988), 137-173, Lecture Notes in Math., 1404, Springer, Berlin, 1989. MR 90m:16002

[BG] K. Bongartz, P. Gabriel: Covering spaces in representation-theory. Invent. Math. 65 (1981/82), no. 3, 331-378. MR 84i:16030

[Br] S. Brenner: A combinatorial characterization of finite Auslander-Reiten quivers, (Ottawa, Ont., 1984), 13-49, Lecture Notes in Math., 1177, Springer, Berlin, New York, 1986. MR $87 \mathrm{~m}: 16045$

[C] J. Clark: Auslander-Gorenstein rings for beginners. International Symposium on Ring Theory (Kyongju, 1999), 95-115, Trends Math., Birkhauser Boston, Boston, MA, 2001. MR 2002f:16010

[CR] C. W. Curtis, I. Reiner: Methods of representation theory. Vol. I. With applications to finite groups and orders. Reprint of the 1981 original. Wiley Classics Library. A WileyInterscience Publication. John Wiley \& Sons, Inc., New York, 1990. MR 90k:20001

[DK] Y. A. Drozd, V. V. Kiričenko: The quasi-Bass orders. (Russian) Izv. Akad. Nauk SSSR Ser. Mat. 36 (1972), 328-370. MR 46:3546

[DKR] Y. A. Drozd, V. V. Kiričenko, A. V. Roŭter: Hereditary and Bass orders. (Russian) Izv. Akad. Nauk SSSR Ser. Mat. 311967 1415-1436. MR 36:2608

[FGR] R. M. Fossum, P. Griffith, I. Reiten: Trivial extensions of abelian categories. Homological algebra of trivial extensions of abelian categories with applications to ring theory. Lecture Notes in Mathematics, Vol. 456. Springer-Verlag, Berlin, New York, 1975. MR 52:10810 
[GM] E. L. Green, R. Martinez Villa: Koszul and Yoneda algebras. Representation theory of algebras (Cocoyoc, 1994), 247-297, CMS Conf. Proc., 18, Amer. Math. Soc., Providence, RI, 1996. MR 97c:16012

[Ha] D. Happel: Triangulated categories in the representation theory of finite-dimensional algebras. London Mathematical Society Lecture Note Series, 119. Cambridge University Press, Cambridge, 1988. MR 89e:16035

[H1] M. Hoshino: Tilting modules and torsion theories. Bull. London Math. Soc. 14 (1982), no. 4, 334-336. MR 83h:16042

[H2] M. Hoshino: On dominant dimension of Noetherian rings. Osaka J. Math. 26 (1989), no. 2, 275-280. MR 90k:16033

[HPR] D. Happel, U. Preiser, C. M. Ringel: Vinberg's characterization of Dynkin diagrams using subadditive functions with application to DTr-periodic modules. Representation theory, II (Proc. Second Internat. Conf., Carleton Univ., Ottawa, Ont., 1979), pp. 280-294, Lecture Notes in Math., 832, Springer, Berlin, 1980. MR 82g:16027

[HN] H. Hijikata, K. Nishida: Bass orders in nonsemisimple algebras. J. Math. Kyoto Univ. 34 (1994), no. 4, 797-837. MR 96a:16012

[I1] O. Iyama: A generalization of rejection lemma of Drozd-Kirichenko. J. Math. Soc. Japan 50 (1998), no. 3, 697-718. MR 99h:16029

[I2] O. Iyama: Some categories of lattices associated to a central idempotent. J. Math. Kyoto Univ. 38 (1998), no. 3, 487-501. MR 2000d:16011

[I3] O. Iyama: $\tau$-categories I: Ladders, to appear in Algebras and Representation Theory.

[I4] O. Iyama: $\tau$-categories II: Nakayama pairs and rejective subcategories, to appear in Algebras and Representation Theory.

[I5] O. Iyama: $\tau$-categories III: Auslander orders and Auslander-Reiten quivers, to appear in Algebras and Representation Theory.

[I6] O. Iyama: Representation theory of orders. "Algebra - Representation Theory" (Edited by K. W. Roggenkamp, M. Stefanescu), Nato-Science-Series, Kluwer Academic Publishers, 63-96 (2001) MR 2002g:16023

[I7] O. Iyama: A proof of Solomon's second conjecture on local zeta functions of orders, J. Algebra 259 (2003), no. 1, 119-126. MR 2003m:11199

[I8] O. Iyama: Finiteness of Representation dimension, Proc. Amer. Math. Soc. 131 (2003), no. 4, 1011-1014. MR 2003k:16024

[I9] O. Iyama: Symmetry and Duality on $n$-Gorenstein rings, J. Algebra 269 (2003), no. 2, 528-535.

[IT1] K. Igusa, G. Todorov: Radical layers of representable functors. J. Algebra 89 (1984), no. 1, 105-147. MR 86f:16029a

[IT2] K. Igusa, G. Todorov: A characterization of finite Auslander-Reiten quivers. J. Algebra 89 (1984), no. 1, 148-177. MR 86f:16029b

[IT3] K. Igusa, G. Todorov: A numerical characterization of finite Auslander-Reiten quivers. Representation theory, I (Ottawa, Ont., 1984), 181-198, Lecture Notes in Math., 1177, Springer, Berlin, 1986. MR 87i:16058

[Ro] K. W. Roggenkamp: Lattices over orders. II. Lecture Notes in Mathematics, Vol. 142 Springer-Verlag, Berlin, New York 1970. MR 44:247b

[R1] W. Rump: Almost abelian categories. Cahiers Topologie Geom. Differentielle Categ. 42 (2001), no. 3, 163-225. MR 2002m:18008

[R2] W. Rump: *-modules, tilting, and almost abelian categories. Comm. Algebra 29 (2001), no. 8, 3293-3325. MR 2002f:18020

[R3] W. Rump: Ladder functors with an application to representation-finite Artinian rings. An. Ştiinţ. Univ. Ovidius Constanţa Ser. Mat. 9 (2001), no. 1, 107-123. MR 2004a:16024

[R4] W. Rump: Lattice-finite rings, to appear in Algebras and Representation Theory.

[R5] W. Rump: The category of lattices over a lattice-finite ring, to appear in Algebras and Representation Theory.

[RV] I. Reiten, M. Van den Bergh: Two-dimensional tame and maximal orders of finite representation type. Mem. Amer. Math. Soc. 80 (1989). MR 90b:16009

[RVo] C. M. Ringel, D. Vossieck, Hammocks, Proc. London Math. Soc. (3) 54 (1987), 216-246. MR 88f:16025 
[S] D. Simson: Linear representations of partially ordered sets and vector space categories. Algebra, Logic and Applications, 4. Gordon and Breach Science Publishers, Montreux, 1992. MR 95g:16013

[T] H. Tachikawa: Quasi-Frobenius rings and generalizations. Lecture Notes in Mathematics, Vol. 351. Springer-Verlag, Berlin, New York, 1973. MR 50:2233

Department of Mathematics, Himeji Institute of Technology, Himeji, 671-2201, Japan

E-mail address: iyama@sci.himeji-tech.ac.jp 